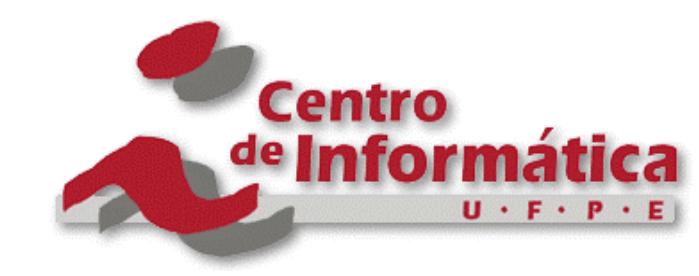

Pós-Graduação em Ciência da Computação

\title{
"THE DEVELOPMENT OF A HARDWARE ABSTRACTION LAYER GENERATOR FOR SYSTEM-ON-CHIP FUNCTIONAL VERIFICATION"
}

Por

\section{TIAGO SAMPAIO LINS}

Dissertação de Mestrado

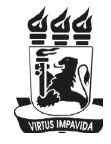

Universidade Federal de Pernambuco posgraduacao@cin.ufpe.br www.cin.ufpe.br/ posgraduacao 


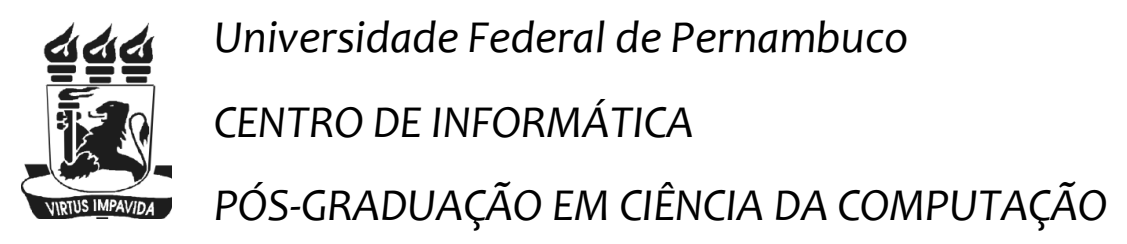

\section{Tiago Sampaio Lins}

\section{"The development of a hardware abstraction layer generator for system-on-chip functional verification"}

Este trabalho foi apresentado à Pós-Graduação em Ciência da Computação do Centro de Informática da Universidade Federal de Pernambuco como requisito parcial para obtenção do grau de Mestre em Ciência da Computação.

ORIENTADOR(A): Profa. Edna Natividade da Silva Barros

RECIFE, FEVEREIRO/2009 
Lins, Tiago Sampaio

The development of a hardware abstraction layer generator for system-on-chip functional verification / Tiago Sampaio Lins. - Recife: O Autor, 2009.

vii., 83 folhas : Il., fig., tab.,

Dissertação (mestrado) - Universidade Federal de Pernambuco. CIn. Ciência da Computação, 2009.

Inclui bibliografia e apêndice.

1. Microeletrônica. 2. Circuitos semicondutores. I. Título.

621.381 CDD (22. ed.) MEI2009- 105 
Dissertação de Mestrado apresentada por Tiago Sampaio Lins à Pós-Graduação em Ciência da Computação do Centro de Informática da Universidade Federal de Pernambuco, sob o título "The Development of a Hadware Abstraction Layer Generator for System-onchip Functional Verification", orientada pela Profa. Edna Natividade da Silva Barros e aprovada pela Banca Examinadora formada pelos professores:

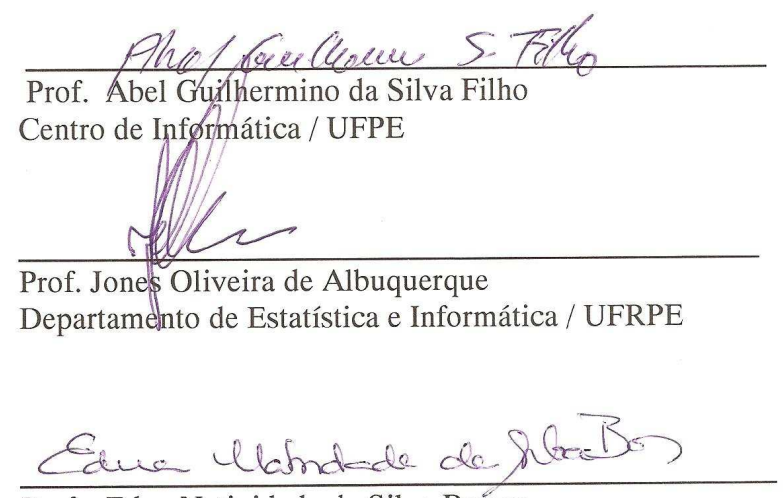

Profa. Edna Natividade da Silva Barros

Centro de Informática / UFPE

Visto e permitida a impressão.

Recife, 17 de fevereiro de 2009.

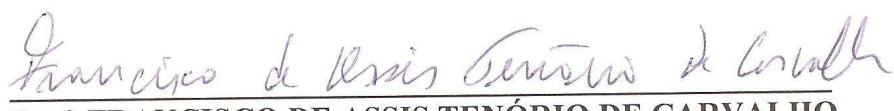

Prof. FRANCISCO DE ASSIS TENÓRIO DE CARVALHO

Coordenador da Pós-Graduação em Ciência da Computação do

Centro de Informática da Universidade Federal de Pernambuco. 


\begin{abstract}
Nowadays functional verification of large system-on-chip has taken about $70 \%$ to $80 \%$ of the total design effort. The large amount of IP's of current SoC's makes the work of verification engineers quite hard due to the need to guarantee that the design is bug free before it is sent to tape out. In order to reduce the time spent in the functional verification and aid the verification engineers with their job, this work presents a Hardware Abstract Layer generator - HAL generator. The HAL generator is part of a methodology for SoC functional verification in development in STMicroelectronics. Such methodology is based on a typical verification flow and supported by IP-XACT; it aims automate tasks in the SoC functional verification flow. To handle these tasks some tools are needed; the HAL generator is one of these tools, being in charge of creating $\mathrm{C}$ functions that allow the manipulation of registers and their fields in a high level way. The HAL functions work as an API in $\mathrm{C}$ to access all their field present in a design, allowing the verification engineers to write their test cases without need to without worrying about masks, macros, define and/or pointers manipulation.
\end{abstract}

Keywords: Functional Verification, System-on-chip (SoC), The Spirit Consortium, Hardware Abstraction Layer (HAL), IP-XACT, Tight Generator Interface (TGI), Automatic Code Generation, $\mathrm{C}$ test cases. 


\section{Resumo Estendido}

Atualmente o processo de verificação funcional de "System-on-Chip" (SoC) complexos envolve centenas de engenheiros ao longo do projeto de um circuito integrado. Tal esforço visa garantir um nível de confiança satisfatório antes do mesmo ser enviado para o "tapeout”. Estudos recentes revelaram que a etapa de verificação funcional constitui de $70 \%$ a $80 \%$ do esforço total do projeto sendo a etapa mais onerosa em termos de custo e tempo dentro do fluxo de desenvolvimento de um SoC.

Devido às restrições de "time-to-market" e a atual complexidade dos SoCs, as equipes de verificação funcional não usufruem do tempo necessário para obter o conhecimento do sistema a ser verificado em profundidade. Contudo eles precisam garantir que todos os módulos de propriedades intelectual (IP) presentes em um SoC continuam funcionando de acordo com a sua especificação após integrados.

$\mathrm{Na}$ verificação funcional existem várias técnicas de como proceder com a mesma. Atualmente, a mais utilizada é a verificação com auxílio de um processador - "processor driven tests”. Dado que nesses complexos sistemas sempre há um processador o qual é responsável pelo controle do sistema. Uma forma de verificar se todos os IPs dentro da hierarquia do SoC funcionam de acordo com suas especificações é executar aplicações em software neste processador e verificar a saída do sistema. A idéia consiste em estimular um IP através de rotinas em software comparando o resultado gerado com o esperado. Nesta abordagem, um dos problemas existente consiste na escrita dos casos de testes. Uma vez que nesses sistemas encontramos uma grande quantidade de IPs, e estando os mesmos dispostos em diferentes níveis de hierarquia de barramentos dentro do $\mathrm{SoC}$, acessar suas interfaces e seus componentes internos utilizando uma linguagem estruturada, como $\mathrm{C}$, é bastante complexo.

Os IPs são acessados via dispositivo de entrada e saída baseado em memória, ou seja, cada IP possui um ou mais endereços e a decodificação desses endereços é feita pelos barramentos existentes no sistema. Para o processador é transparente se uma rotina de escrita ou leitura vai ser processada por um bloco de memória de fato ou por um IP com um conjunto de registradores internos. Desta forma, quando o engenheiro de verificação está escrevendo os casos de testes ele precisa lidar com manipulação de endereços, macros e/ou estruturas, defines, ou ainda, partir para uma linguagem de montagem. 
No processo de escrita dos casos de testes, as rotinas que implementam o acesso aos registradores e seus campos consistem em uma atividade fundamental para permitir o teste de funcionalidade do sistema. A essas rotinas encarregadas de ler e escrever valores nos registradores internos do IPs de um SoC chamamos de "camada de abstração de hardware" - "Hardware Abstraction Layer" (HAL).

A STMicroelectronics, uma das lideres mundial na industria de semicondutores, através do seu grupo de verificação funcional decidiu desenvolver uma metodologia para verificação funcional de SoC que fizesse o uso de ferramentas específicas para automatizar parte das atividades relacionadas a esta fase do projeto de um circuito integrado. Uma necessidade do time de verificação funcional, no contexto desta metodologia, consistia em possuir uma ferramenta capaz de automatizar o processo de geração dessa HAL, uma vez que os engenheiros de verificação tinham bastante trabalho na escrita dessas rotinas e em muitos casos elas eram responsáveis por falhas no processo de verificação funcional. $\mathrm{O}$ interesse do time era permitir que o engenheiro focasse seu trabalho no teste de funcionalidade do SoC não despendendo seu tempo na implementação das rotinas de acesso aos registradores dos IPs.

Este trabalho foi desenvolvido em parceira com a "STMicroelectronics" visando estudar e propor uma ferramenta capaz de atender tal necessidade. O trabalho propõe a implementação de uma ferramenta capaz de gerar a camada de abstração de hardware.

A ferramenta proposta, "HAL generator", é um dos instrumentos que suportam a automação de parte do processo de verificação funcional. Esta ferramenta é responsável pela geração automática de uma API que permite a manipulação dos valores dos registradores. A manipulação dos valores pode ser feita em todo registrador ou em subcampos do mesmo através de uma interface em um alto nível de abstração. Os engenheiros de verificação utilizam as funções geradas pelo "HAL generator" para ler e escrever valores nos registradores do $\mathrm{SoC}$ a ser verificado.

A ferramenta é capaz de gerar tanto a declaração como definição das funções. As funções são geradas na linguagem de programação C e funcionam como uma API para ler e escrever valores nos registradores. Com o apoio dessas funções geradas pela ferramenta, a codificação dos casos de testes fica mais fácil e produtiva, permitindo ainda a reutilização dos casos de testes em outros sistemas dado que a API gerada pelo "HAL generator" segue um padrão na geração das funções o qual garante que um caso de teste 
para um mesmo IP possa ser executado em contextos diferentes, bastando apenas executar o "HAL generator" para cada cenário. 


\section{Table of Contents}

1. INTRODUCTION ................................................................................................................................................12

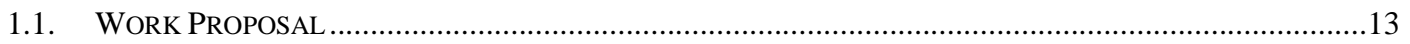

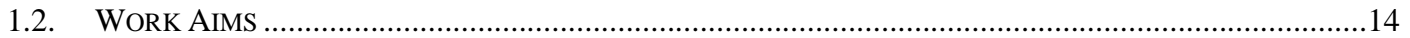

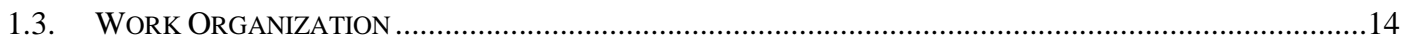

2. FUNCTIONAL VERIFICATION: STATE OF THE ART .....................................................................16

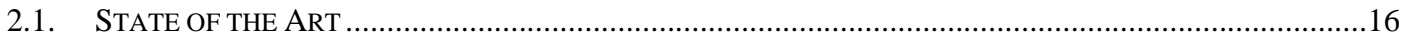

2.1.1. Hardware Description Language (HDL) Testbenches .......................................................... 17

2.1.2. Processor Driven Testbenches (PDT) ........................................................................ 18

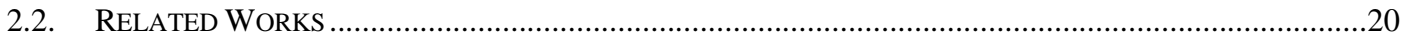

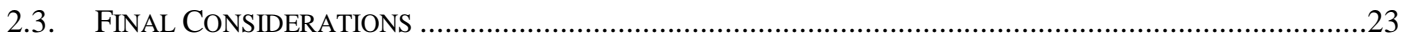

3. SYSTEM-ON-CHIP FUNCTIONAL VERIFICATION: SPRIT ASSISTED VERIFICATION

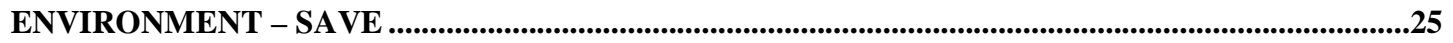

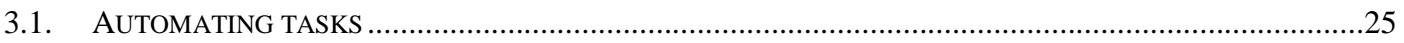

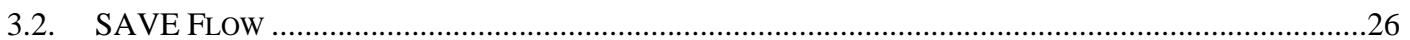

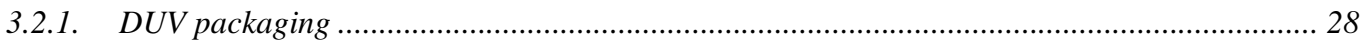

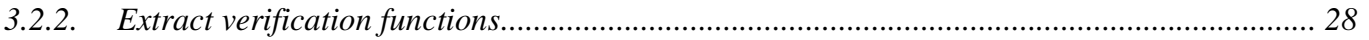

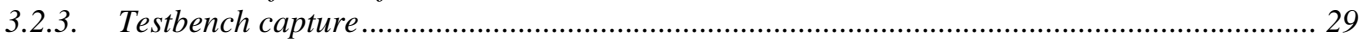

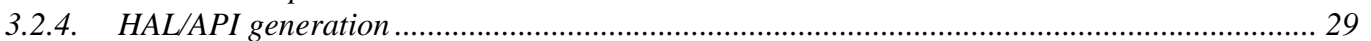

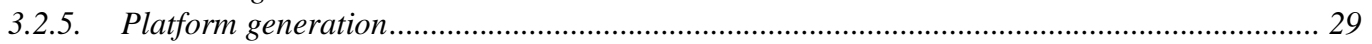

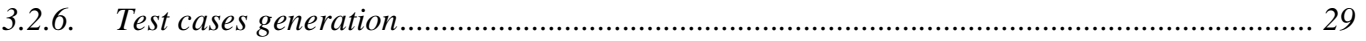

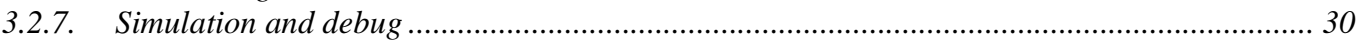

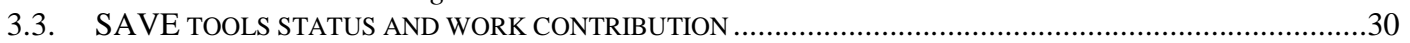

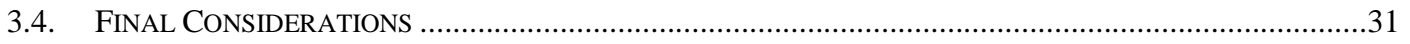

4. THE PROPOSED HARDWARE ABSTRACT LAYER (HAL) GENERATOR ....................................32

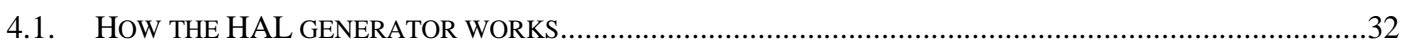

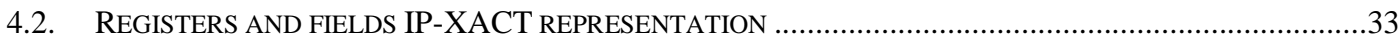

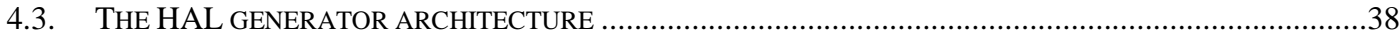

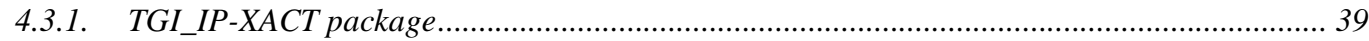

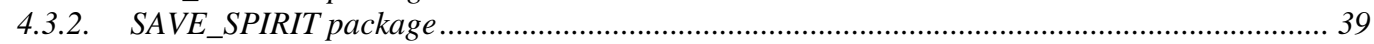

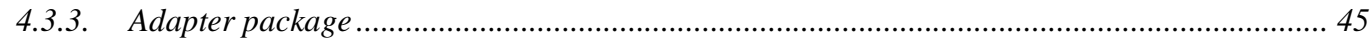

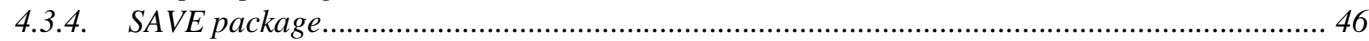

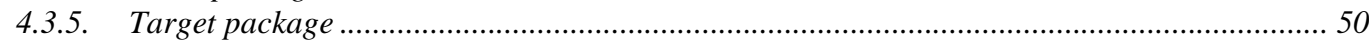

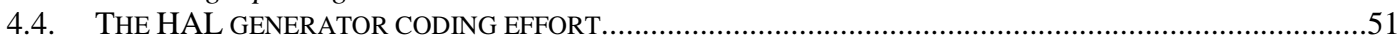

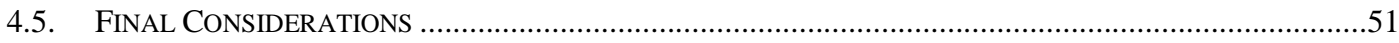

5. CASE STUDY - USING THE HAL GENERATOR IN A SOC FUNCTIONAL VERIFICATION ........53

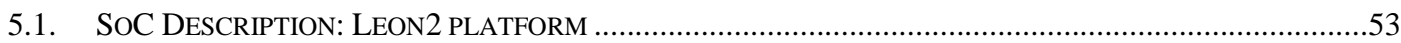

5.2. UNIVERSAL ASYNCHRONOUS RECEIVER/TRANSMITTER (UART) MODULE.................................54

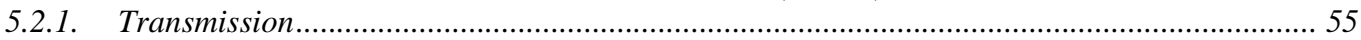

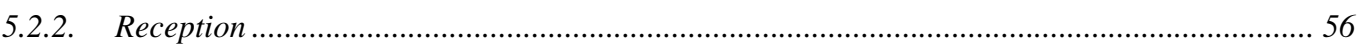

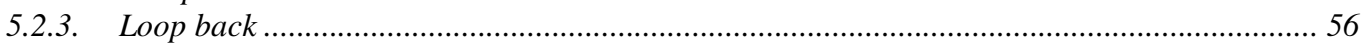

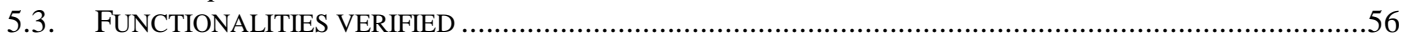

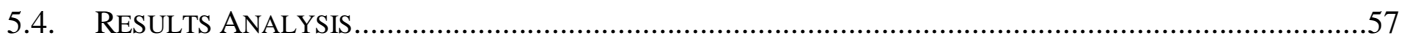

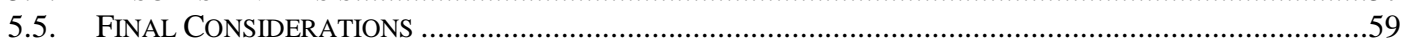

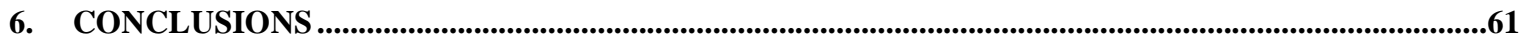

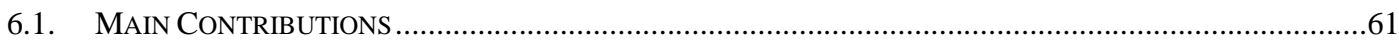

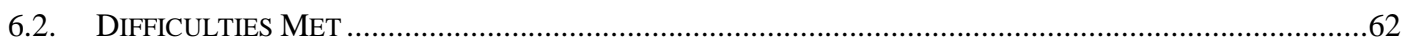

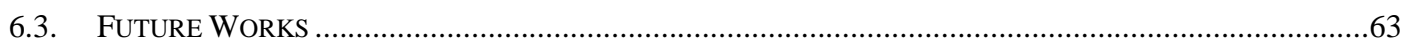

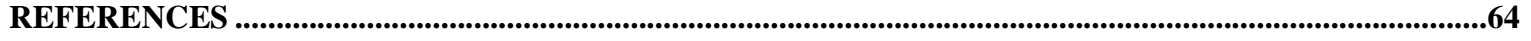

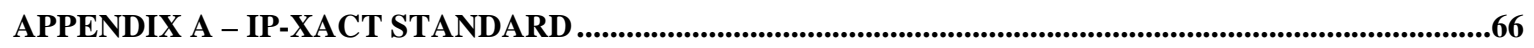

APPENDIX B - SLAVE INTERFACES BASE ADDRESS DEFINITION ALGORITHM ENGINE............77

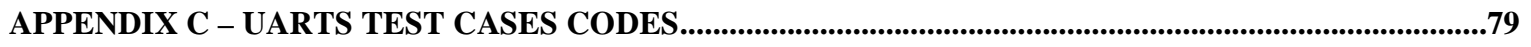




\section{List of tables}

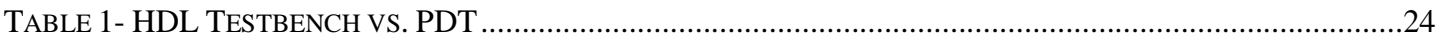

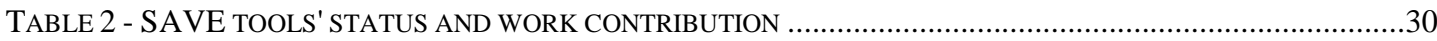

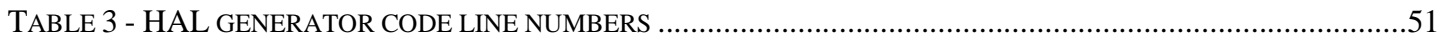




\section{List of figures}

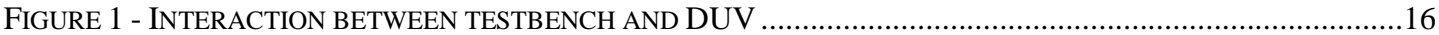

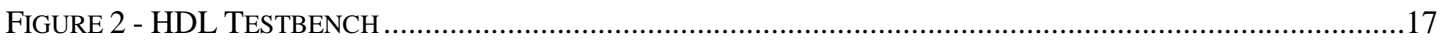

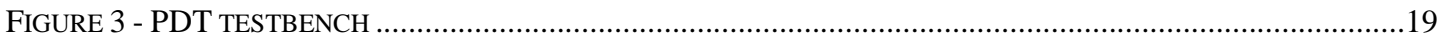

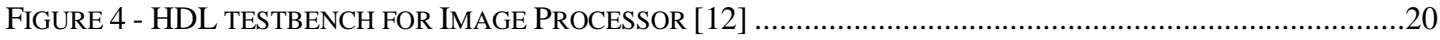

FIGURE 5 - PROCESSOR DRIVEN TESTBENCH FOR IMAGE PROCESSOR [12] ............................................21

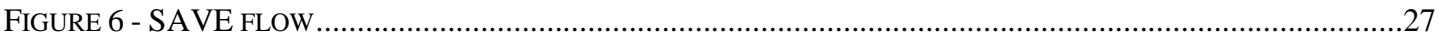

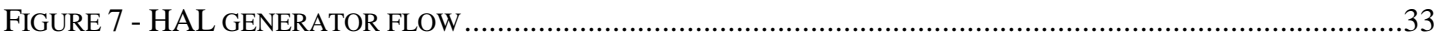

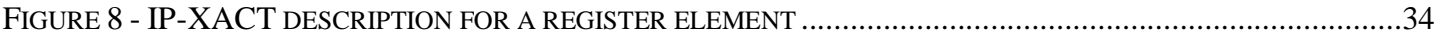

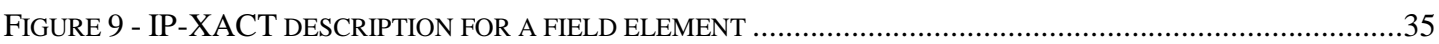

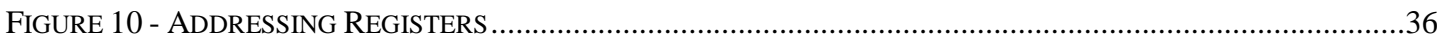

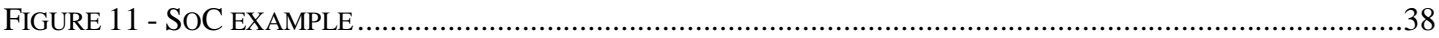

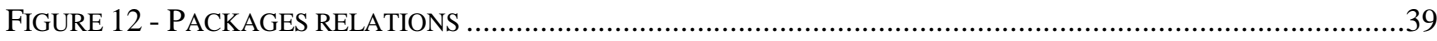

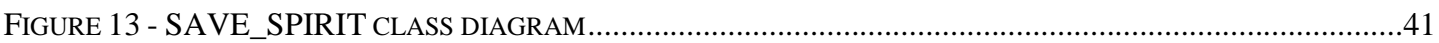

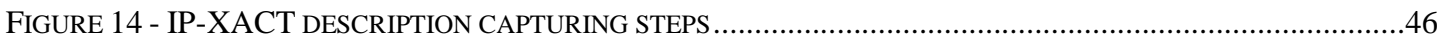

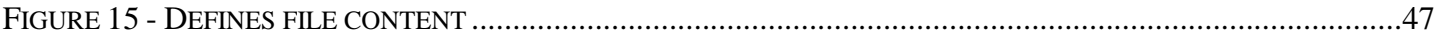

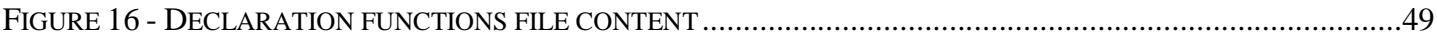

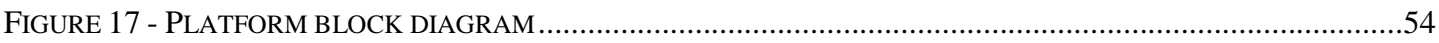

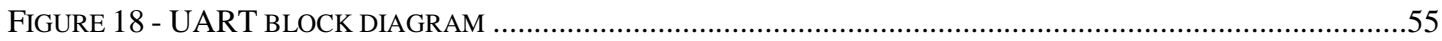

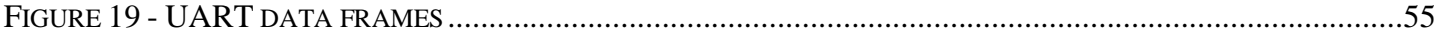

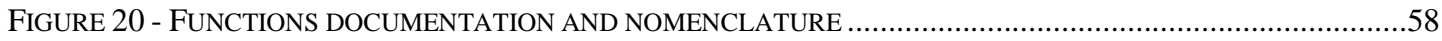

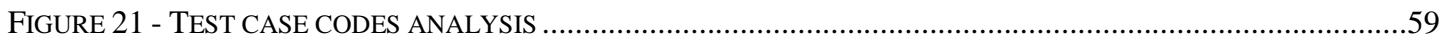

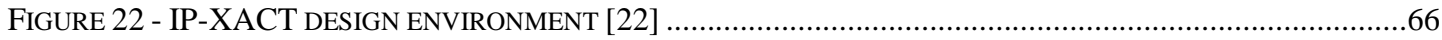

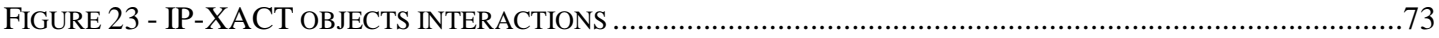

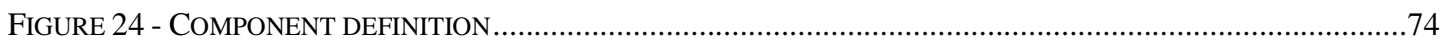

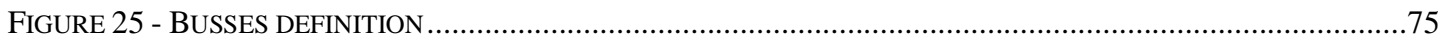

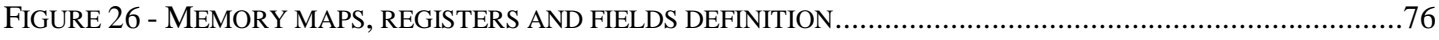

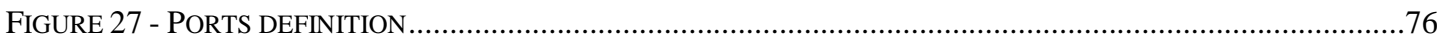

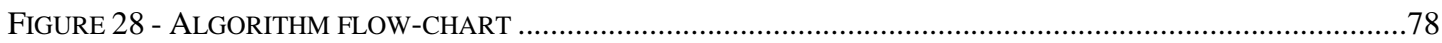




\section{Definitions, Acronyms and Abbreviations}

For the purposes of this document, the following terms and definitions apply. 


\section{Definitions}

Design Environment (DE): The coordination of a set of tools and IPs, the systemdesign and implementation flows of a SoC reuse environment. This is managed by creating and maintaining a meta-data description of the SoC.

Design Under Verification (DUV): IP, sub-system or SoC object of investigation of the functional verification.

Extensible Markup Language (XML): A general-purpose specification language. It is classified as an extensible language because it allows its users to define their own elements. Its primary purpose is to help information systems to share structured data.

Electronic Design Intellectual Property (IP): This is a term used in the electronic design community to refer to a reusable collection of design specifications which represents the behavior, properties, and/or representation of the design in various media. The name IP is partially derived from the common practice of considering a collection of this type to be the intellectual property of one party. Both hardware and software collections are encompassed by this term. IP is utilized in the context of a SoC design.

Functional Verification: Task of verifying if an electronic system design conforms to specification.

Hardware Abstraction Layer (HAL): An abstraction layer, implemented in software, between the physical hardware and the software that runs on the CPU.

IP-XACT: Defines an XML meta-data and schema model. IP-XACT was created by The SPIRIT Consortium as a standard for describing and handling intellectual property that enables automated configuration and integration through tools.

Register Transfer Level (RTL): A way of describing the operation of a synchronous digital circuit. In RTL design, a circuit's behavior is defined in terms of the flow of signals (or transfer of data) between hardware registers, and the logical operations performed on those signals.

System-on-Chip (SoC): Refers to integrating all components of an electronic system into a single integrated circuit (chip). It may contain digital, analog, mixed-signal, and/or often radio-frequency functions all on one chip. 
Spirit Assisted Verification Environment (SAVE): A functional verification flow for an IP, sub-system or SoC based on IP-XACT standard features.

Tight Generator Interface (TGI): Used to manipulate values of elements and attributes for IP-XACT compliant XML.

Transaction-Level Modeling (TLM): An abstraction level higher than register transfer level (RTL), used for specifying, simulating, verifying, implementing, and evaluating SoC designs. 


\section{Acronyms and abbreviations}

AHB AMBA high speed bus

AMBA Advanced Microcontroller Bus Architecture

APB AMBA peripheral bus

API Application Programmer Interface

DE Design Environment

DMA Direct Memory Access

DUT Design Under Test

DUV Design Under Verification

HAL Hardware Abstraction Layer

IDE Integrated Development Environment

IP Electronic Design Intellectual Property

SAVE Spirit Assisted Verification Environment

SoC System on Chip

SPIRIT Structure for Packaging, Integrating and Re-using IP within Tool-flows

TGI Tight Generator Interface

UML Unified Modeling Language

XML Extensible Markup Language 


\section{Introduction}

Today's verification of large system-on-chip (SoC) designs is an extremely complex process involving hundreds of man months of work to reach a sufficient confidence level for tape out [4]. According [19] the authors say that verification today constitutes about $70 \%$ to $80 \%$ of the total design effort, thereby making it the most expensive activity in terms of cost and time, in the entire design flow.

Before a system-on-chip (SoC) be sent to tape out, the functionality and the connections of each sub-system and IP have to be verified. This is one of the SoC verification engineers' roles. They write a set of test cases that verify if the IP is still functional as intended by the design engineer.

Due to time to market constraints and the complexity of new SoC, the functional verification team cannot gain a deep understanding of the target design [25]. They need to guarantee that all IPs present in a SoC are still functional as intended by the design engineer, even without the deep understanding of the system. To manage this situation new techniques and methodologies are constantly researched in the functional verification.

Processor Driven Tests (PDTs) are test vectors driven into the design via the processor bus and can originate from several types of processor models. For designs that incorporate or interface to an embedded CPU, processor driven tests can be a valuable addition to the suite of tests used to perform functional verification. With a full functional model of the processor, tests in the form of embedded code are written in $\mathrm{C}$ or assembly and are compiled to the target processor. These tests more accurately replicate design function where the processor comes out of reset and begins fetching instructions which result in reads and writes to registers of peripherals, IP or custom logic.

In the processor driven tests the design runs the system like it is delivered in the end product, reading the application from the source memory, processing and storing or outputting the result. In this way the processor driven test more accurately replicates the designs operation, testing not only the IP functionality but its interface to the sources and destinations as well as the ability of the CPU to communicate with all devices present in the design.

One challenge in PDTs consists in write $\mathrm{C}$ or assembly test cases. Due to the low level of the registers in a SoC hierarchy to access their value we need to manage assembly routines and/or work with many macros and defines. Generally the verification engineer 
needs to write those codes by hand; a step in functional verification very susceptive to error due to the complexity of write assembly and/or macros codes, in addition, manage defines and pointers structures.

During the functional verification of complex SoC the test case coding became even harder due to the different bus level hierarchy and the amount of registers and their fields found on it. Each field has it own access police, it means that we can read-only, write-only or read/write values to the field. For instance, writing a value to a read-only register could invalidate the test due to it violation can force an unexpected behavior of the design under verification. When a register is placed in a low-level bus hierarchy in the SoC the verification engineer needs to check each bridge and channel present in the bus hierarchy to understanding the address hierarchy and find each register address. A complex work is accessing the registers and defining how they should be stimulated considering their relations with other registers in order to verify that the system works as specified. The test case coding is a critical phase during the functional verification; increases the success criteria in that step meaning increase the chances of success of the project.

Under this scenario the Functional Verification Group (FVG) from STMicroelectronics [20] decide put in place a methodology to reduce the effort and time spent in the SoC functional verification. STMicroelectronics was created in 1987 by the merger of SGS Microelettronica of Italy and Thomson Semiconducteurs of France, ST today is one of the world's largest semiconductor companies. The Functional Verification Group is in charge of the development and use of solutions for functional verification and validation that aim to minimize the time-to-market and maximize productivity for key projects in ST. Among others obligations, the FVG is responsible to representing ST in some standardization activities around the world, such as supporting the SPIRIT [21] Verification Working Group. The FVG proposed a methodology called Spirit Assisted Verification Environment that aims to automate tasks present in the functional verification flow. In order to make it feasible some tools are needed to handle these tasks; one of these tools is the Hardware Abstract Layer generator. This master research consist in investigate how the Hardware Abstract Layer generator can be implemented.

\subsection{Work Proposal}

This work investigates and proposes an implementation model of a Hardware Abstraction Layer (HAL) generator; a tool that is part of the Spirit Assisted Verification Environment (SAVE). The SAVE defines a functional verification flow and is supported 
by the IP-XACT standard [22] from The Spirit Consortium [21]. The IP-XACT is a standard for describing and handling IPs and designs that enables automated configuration and integration through tools. The main objective of SAVE is automating tasks in the SoC functional verification flow aiming reduce the effort and the time spent in that process. SAVE uses the IP-XACT description of a SoC to capture information regarding its contents; and uses it in order to generate testbenches, hardware abstractions functions, and simulation platforms in an automatic way supported by tools.

The HAL generator is one of those tools present in SAVE that support the functional verification process automation. This tool is responsible to generate functions that allow the register and their fields' manipulation in a high level abstraction. Verification engineers use the HAL functions during the test case coding to set and get the values of the registers found in a SoC. The functions are generated in C language and work as an API to read and write values to the registers. With the support of the hardware abstraction functions, $\mathrm{C}$ test case coding becomes easier, more productive and reusable as the verification engineers don't need to write their own routines to access the registers and their fields by hand.

\subsection{Work Aims}

This work intend improve the SAVE methodology implementing the Hardware Abstract Layer generator. The aims of the work are:

- Investigating how the tool can be implemented;

- Propose an architecture and implementation model for the tool;

- Produce initials results about the effort and time spent in the SoC functional verification when supported by HAL functions.

\subsection{Work Organization}

The others Sections of this work are structured in following manner:

- Section 2: presents a brief description of the start of the art in functional verification and discuss the related works;

- Section 3: presents the SAVE methodology and this master research contribution inside of the methodology; 
- Section 4: describes how the HAL generator works and interacts with the IPXACT description. This Section also presents the architecture proposed and some numbers regarding the tool implementation;

- Section 5: discuss a case study of a SoC functional verification using the HAL generator and given a result analysis;

- Section 6: presents the conclusions of this work, where are describe the main contributions, difficulties met and future works. 


\section{Functional Verification: State of the Art}

This Section aims describe the state of the art regarding functional verification methods. Furthermore, brings the recently studies and works related with System-on-Chip functional verification.

\subsection{State of the Art}

Functional Verification is the process used to demonstrate that the intent of a design is preserved in its implementation. It means that the functional verification proposal is guarantee that the results of the implementation meet the specification [9].

In the context of functional verification the word testbench is used to represents the code written to build the environment to verify the design. This environment consists of structures to create a sequence of predefined stimulus to supply a such design and to check the output. Also are part of the testbench structures to allow the data communication and control. Figure 1 illustrates how the testbench interact with the design under verification (DUV), also referenced as design under test (DUT).

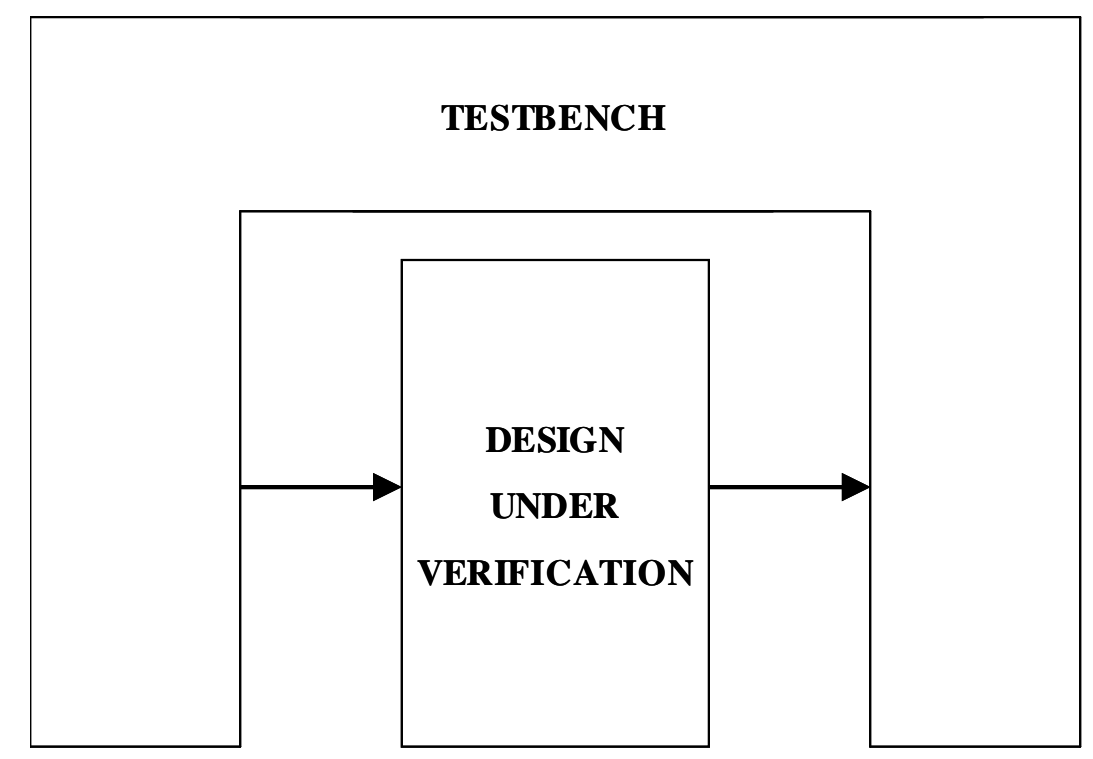

Figure 1 - Interaction between testbench and DUV

In sequence the two functional verification methods most researched and used in academic and industry context in lately are presented. Both were used as theoretical basis for this master research. 


\subsubsection{Hardware Description Language (HDL) Testbenches}

HDL testbenches [9] [10] [11] has been used along the years as the traditional functional verification method. In this method stimulus is provided to exercise each line in the IP or sub-system HDL codes. A testbench is built to verify the functionalities of the design by providing meaningful scenarios to check that given certain input, the design performs according the specification. A HDL testbench environment is composed of several types of components that can be seen in Figure 2. Each component is detailed in following.

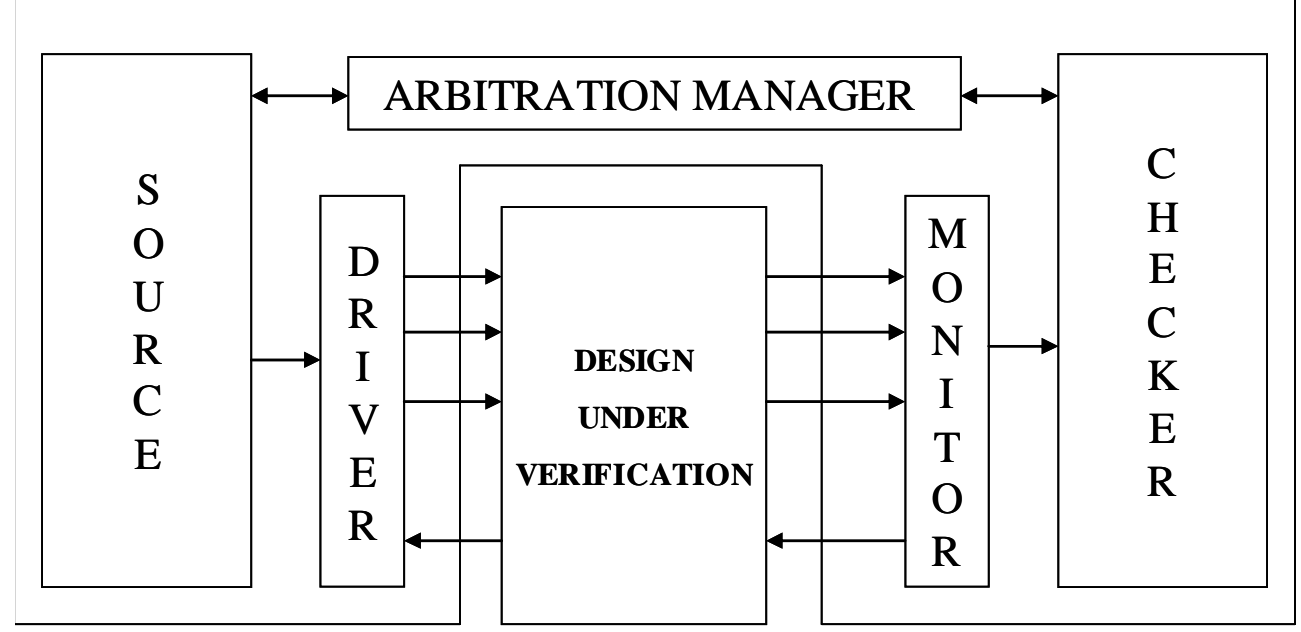

Figure 2 - HDL Testbench

- The source generates input vectors. Modern sources generate random, biased, and valid stimuli. Sources also bias the stimuli toward design corner cases to further stress the logic. Biasing and randomness serve different goals and there are tradeoffs between them, hence different sources have a different mix of these characteristics;

- The drivers translate the stimuli produced by the generator into the actual inputs for the design under test. Generators create inputs at a high level of abstraction, namely, as transactions or assembly language. The drivers convert this input into actual design inputs as defined in the specification of the design's interface;

- The monitor converts the state of the design and its outputs to a transaction abstraction level so it can be stored in a database to be checked later on;

- The checker validates that the contents of the database are legal. There are cases where the generator creates expected results, in addition to the inputs. In these cases, the checker must validate that the actual results match the expected ones; 
- The arbitration manager manages all the above components together. It can be configured to stop the verification process if an error is detected by the checker module;

- The design under verification consists of the module intent to be verified.

The use of HDL testbenches is most recommended in an IP functional verification context due to an IP generally has a specific functionality against many functions of a SoC. A DUV with several functionalities implies a more complex source in consequence more effort to build it. Once the role of the source is generate the stimulus that will verify the DUV even more functionalities its presents more complex the process to build the source. Using a HDL testbench the design can use the same methodology to verify the DUV from HDL implementation level until FPGA prototype.

\subsubsection{Processor Driven Testbenches (PDT)}

Processor driven testbenches (PDT) are test vectors (stimulus) driven into the design under verification via a processor bus and can originate from several types of processor models. For designs that incorporate or interface to an embedded CPU, PDT can be a valuable strategy to perform functional verification [2] [7] [12].

In a processor driven tests context we can use a bus functional model or a full processor model to execute a program (executable code) which stimulates the design under verification. For a bus functional model these programs consist of a sequence of reads and writes to various registers or memory locations serviced by the processor bus. With a full processor model, programs in the form of embedded code are written in $\mathrm{C}$ or assembly and are compiled to the target processor. These programs more accurately replicate design function where the processor comes out of reset and begins fetching instructions which result in reads and writes to registers of peripherals, IP or custom logic. Figure 3 illustrates a typically PDT scenario. 


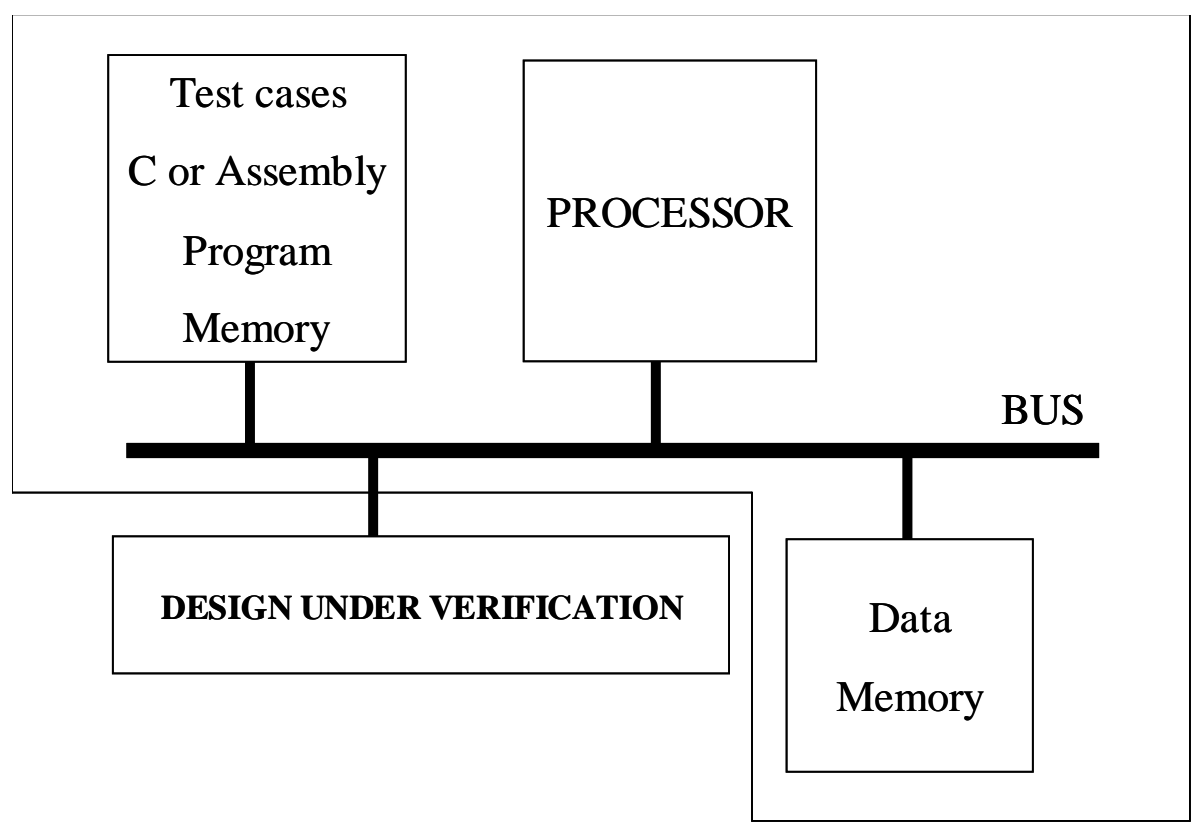

Figure 3 - PDT testbench

- The program memory stores the $\mathrm{C}$ or assembly programs that will be executed by the processor. In case of a testbench built with a bus functional model the program will perform just reads and writes procedures.

- The processor (bus functional model or full processor model) is responsible to execute the $\mathrm{C}$ or assembly program stored in the program memory.

- The bus handles the data communication throughout the testbench components. In case of a bus functional model the bus component heritage the processor role that is the processor component is not present in the testbench. In that case the bus functional model is responsible to handle the program memory.

- The data memory stores intermediary and output values. The results stored in the data memory are used to check the correctness of the functional verification. This checking process is done by routines present in the program stored in the program memory.

- The design under verification consists of the module intent to be verified.

PDT was introduced in functional verification due to the increasing system-on-chip complexity and size. The HDL testbenches face problems of controllability and observability to verify complexes SoCs [7]. The same functional verification methodology can be followed from the HDL implementation until post tape-out phase when PDT is used. 


\subsection{Related Works}

The growing complexity of SoCs has led to a significant increase of verification efforts, imperative to meet the time-to-market demands. In a SoC hierarchy the simple task of checking all bus interconnections becomes fairly complex when there are eighty IP's integrated on the same SoC. Due to this scenario new methods [7] [12] and techniques [3] [13] [18] [24] are being sought aiming to obtain a shorter functional verification time, a desirable level of coverage and a slightly verification flow.

Jim Kenney [12] discuss the processor driven testbench method and presents its strengths and weakness, he also examines the inherent value of combining PDT with traditional HDL testbenches. In his article he utilizes an image processor as a case study and put in place the two methods presents in the state of the art Section of this master thesis to procedure the functional verification of the processor (DUV).

Figure 4 depicts HDL testbench built in [12] connected to the image processor. The HDL Source Data module is responsible to generate the stimulus to the DUV. The AMBA Bus module executes the role of arbitration manager. The HDL Results Checker is responsible to procedure the verification check. The driver and monitor role are accumulated by the HDL Source Data and HDL Results Checker modules respectively. The testbench initializes the image processor registers, feeds it pixels for processing and compares the resultant pixels to the expected output.

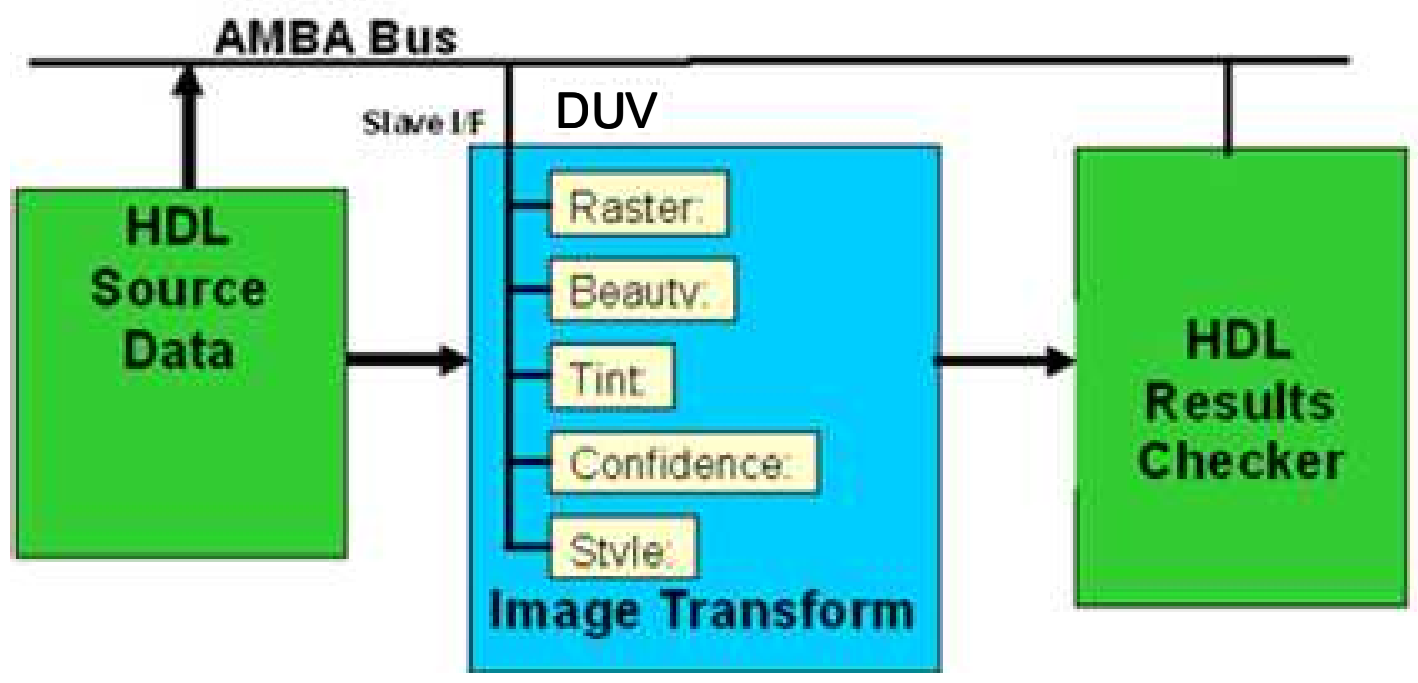

Figure 4 - HDL testbench for Image Processor [12]

In contrast the processor driven test depicted in Figure 5 places the image processor insitu, surrounded by the source and destination memories and interfaced to the processor itself. The test, written in $\mathrm{C}$ and executed by the CPU as embedded code, loads the source 
memory with an image, initializes the image processor and clocks the design until a destination image is produced.

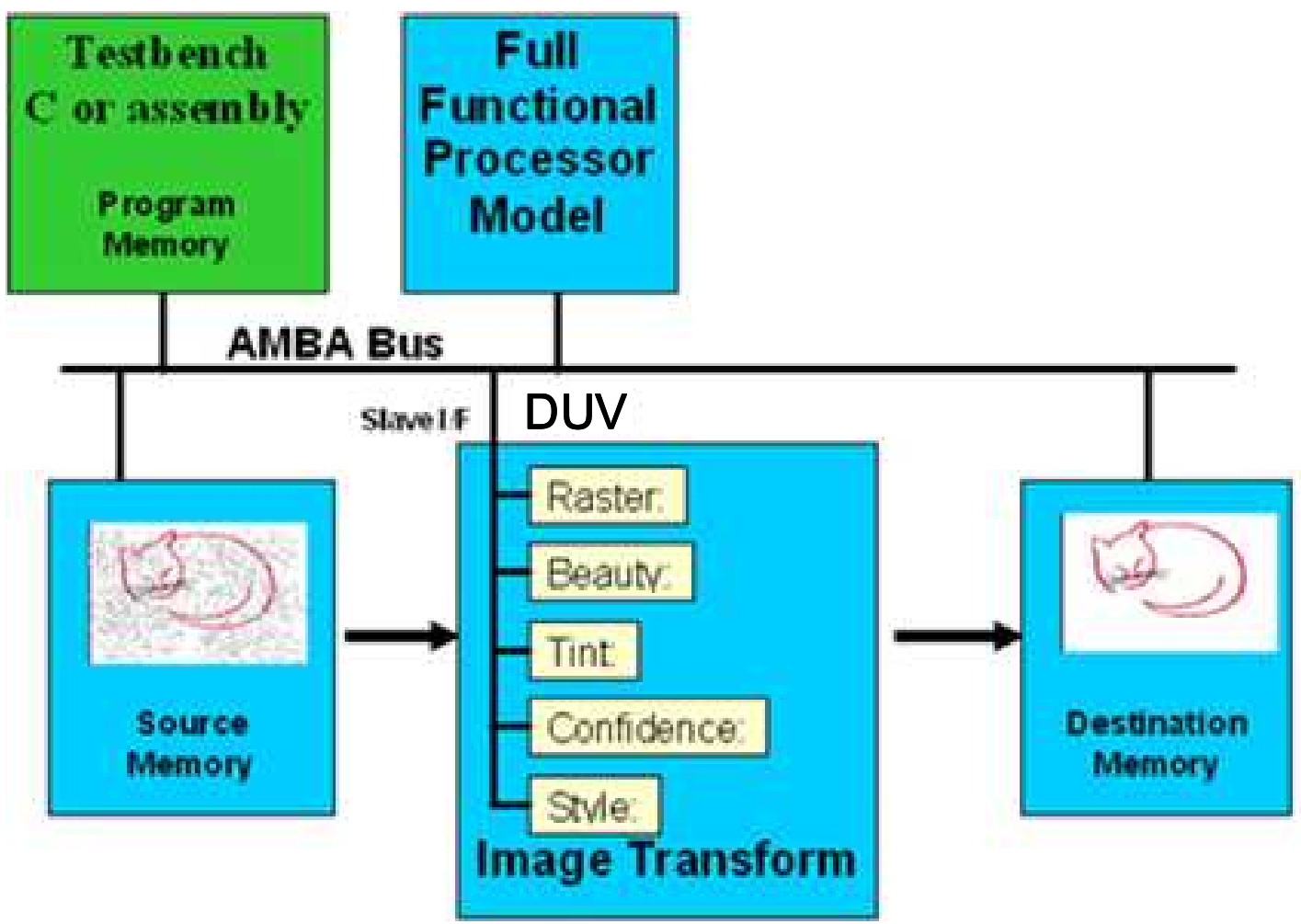

Figure 5 - Processor driven testbench for image processor [12]

Comparing these two methods we can make some considerations. We can notice that once the test is setup, the design runs as it would in the end product, reading the source memory, transforming the image and storing the result in destination memory. In this way the processor driven testbench more accurately replicates the designs operation, testing not only the image processor but its interface to the source and destination memories as well as the ability of the CPU to communicate with all three devices.

PDT also offers gains in reusability. It's not difficult to imagine this block-level processor driven testbench being migrated to the sub-system or SoC level. Assuming additional hardware interfaces are not inserted between the image memories or image processor, the $\mathrm{C}$ code for this test can be reused without change at the sub-system and SoC level. In fact, since the test is driven by the processor, this test can also be run on the live target of an FPGA prototype or the fabricated device itself. In case of a HDL testbench we can only reuse the testbench until FPGA level once the HDL testbench can not be fabricated together with the design.

Arie Komarnitzky [14] shares the same idea of Jim Kenney [12] in their work and presents a functional verification flow for a group of SoC devices that connect several IPs 
from different vendors with customer specific IPs, around an industry standard AMBA bus [1]. The authors demonstrated on several SoC designs that functional verification of complex SoC can be achieved mainly by connectivity and application specific tests written in $\mathrm{C}$. While expensive verification methodologies are necessary to assure full coverage during IP development, their approach to SoC verification targets assurance of system connectivity vs. system specification and correct system behavior under application software that will actually run on the device. The approach presented by Jim Kenney [12] and Arie Kormarnitzky [14] for functional verification of SoC based on PDT is cost effective not only due to faster C code entry vs. RTL, but also because of heavy reuse of application code coming from system SW development for RTL verification purposes.

As we can see processor driven tests attack functional verification of hardware from a unique angle showing advantages and benefits. As such it can detect design errors that can be not found by traditional HDL testbenches. PDT can be easily migrated from block to SoC level verification and across the simulated and physical domains. The method exercises the design in a native fashion and can be applied to any function directly or indirectly accessible by the processor bus.

From [14] and [16] we can see the advantages and benefits of PDT over HDL testbenches method. However, even the PDT shows several enhancements in functional verification, the method also presents points to be improved. One of these points consists in the activity of writing $\mathrm{C}$ test cases.

In [12] and [14] the authors discuss the benefits of processor driven tests method, but they don't address the $\mathrm{C}$ test case coding activity, one step present in that method crucial for the functional verification success. This master research presents a tool which aiming to improve PDT method proposing to automate part of the $\mathrm{C}$ test case coding activity. The idea behind it is generating part of the code needed to access the registers and their fields through a high level API. The tool is a complementary instrument that supports the functional verification process in the activity of writing $\mathrm{C}$ or assembly embedded test programs.

In PDT to achieve the verification there is a need of software layers to interface the C or assembly embedded test programs with the hardware. One of these layers is a Hardware Abstraction Layer (HAL) this layer is specific to the hardware implementation and is made of thin software layers. It mainly includes hardware registers read and write operations [7]. By raising the abstraction level of the test programs, we obtain two main advantages: 
- Decrease their complexity and then reduce the cost of debug;

- Increase reusability, as they are independent from the hardware implementation.

The concept of Hardware Abstraction Layer in the context of SoC design is explained in [24]. The authors define HAL as all the software that is directly dependent on the underlying hardware. The examples of HAL include boot code, context switch code, codes for configuration and access to hardware resources.

In [7] the authors introduce a method that also allows the use of high-level software test programs for SoC functional verification. The key idea behind their work is to write high-level test programs using an API and generating a specialized OS that allow efficient execution of test programs. It is then possible to generate high-level test programs, which are independent from hardware implementation. The authors achieved the success enabling the hardware abstraction but introduced an overhead in terms of debugging and simulation time due to they put in place three hierarchical layers (HAL->API->OS) strategy. In this master research the tool proposed generates the HAL and API interface automatically allowing the access of all hardware resources without the needing of an OS.

\subsection{Final Considerations}

This Section firstly presented the state of the art in functional verification discussing the two most recently used methods, HDL testbench and PDT. The first method is more traditionally being used along the years. It consists to use HDL components to generate stimulus to the DUV and also to checks the output. Nowadays due to the increasing complex of the SoCs the effort to build these HDL components has compromised a long period from SoC development. In this scenario the PDT method comes up gaining importance. The PDT uses $\mathrm{C}$ and assembly embedded test programs running in a CPU or DSP to stimulate the DUV. The process of create complex and varied stimulus for the DUV by writing $\mathrm{C}$ or assembly code is much easier than by HDL method. 
Table 1 presents a comparison between the HDL testbench and PDT.

Table 1- HDL Testbench vs. PDT

\begin{tabular}{|l|l|}
\hline \multicolumn{1}{|c|}{ HDL testbench } & \multicolumn{1}{c|}{ PDT } \\
\hline Most applied in IP verification & Most applied in SoC verification \\
\hline Independent of CPU or DSP & Mandatory the presence of CPU or DSP \\
\hline $\begin{array}{l}\text { Same test cases from implementation until } \\
\text { FPGA prototipation }\end{array}$ & $\begin{array}{l}\text { Same test cases from implementation } \\
\text { until post tape-out. }\end{array}$ \\
\hline
\end{tabular}

In a second moment was discussed related works approaching PDT method and its advantages and benefits over HDL testbench were shown. The process of use C or assembly test programs configure a better alternative for SoC functional verification comparing with traditional testbenches; however in the context of the PDT it is considered a point to be improved. The test programs must be able to manipulate all registers present in the SoC hierarchy in a right manner respecting its access police and also the relation between them. When a $\mathrm{C}$ language is used it means that we need to access hardware resources from a high level of abstraction. To support this activity was introduced the Hardware Abstraction Layer concept which consists utilize software layers to create an abstraction level for the hardware resources providing an easier way to write the test programs.

The next Section presents the Spirit Assisted Verification Environment a SoC functional verification flow, which the tool developed in this master research is inserted. 


\section{System-on-Chip Functional Verification: Sprit Assisted Verification Environment - SAVE}

This Section aims to present the Spirit Assisted Verification Environment (SAVE). SAVE is a System-on-Chip functional verification methodology based on a typical processor driven testbenches method. The main objective of this methodology is automating tasks in the $\mathrm{SoC}$ functional verification flow aiming reduce the time spend during the SoC functional verification phase. To handle some of these tasks SAVE defines a set of tools. One of these tools is the Hardware Abstract Layer generator, which is the subject under investigation in this master research.

The basics concepts behind SAVE methodology are described in this Section to contextualize the reader in the big picture which this master research is inserted. The SAVE strategy to allow the tasks automating and its phases are discussed in the sequence. At the end of this Section the SAVE development status is presented and this master research contribution highlighted.

\subsection{Automating tasks}

One manner to decrease the time spend during the $\mathrm{SoC}$ functional verification is substituting the hand work, like coding test cases, testbenches or building platforms to simulate the design by tools that perform these tasks or part of them [15]. But to make feasible automatize these tasks we need to implement ways to enable the tools to capture the SoC description. In generally the SoC description appears like code lines or in a document written in natural language being complex to design a tool able to introspecting in the SoC description and capture information from it.

Aiming to create a universal manner to describe SoCs and IPs that allow both human readable and machine processable sources the IP-XACT standard [22] was proposed. The IP-XACT standard is managed by The SPIRIT Consortium [21], an industry association of leading companies including ARM, Cadence, LSI, Mentor Graphics, NXP, ST, Synopsys and Texas Instruments and over 50 other reviewing member companies.

The standard describes an eXtensible Markup Language (XML) [6] data format and structure, documented with a schema for capturing the meta-data, which documents the design of the intellectual property used in the development, implementation, and verification of electronic systems. The standard also includes a tight generator interface 
(TGI) to provide consistent, tool-independent access to the meta-data. The XML documents described and validated by the schema comprise a standard method to document IP that is compatible with automated integration techniques.

To allow the tasks automatize the SAVE methodology is supported by IP-XACT. The standard is used during all the SAVE's SoC functional verification flow. How SAVE uses the IP-XACT standard is described in the next Section

The SAVE flow is presented in sequence and its main steps are highlighted. In Appendix A the IP-XACT standard are discussed with more details.

\subsection{SAVE Flow}

Figure 6 shows a diagram with a typical verification flow for an IP, sub-system or SoC that involves the writing of a verification plan, the construction of a testbench, the generation of test case code and the simulating and debugging of the test cases. 


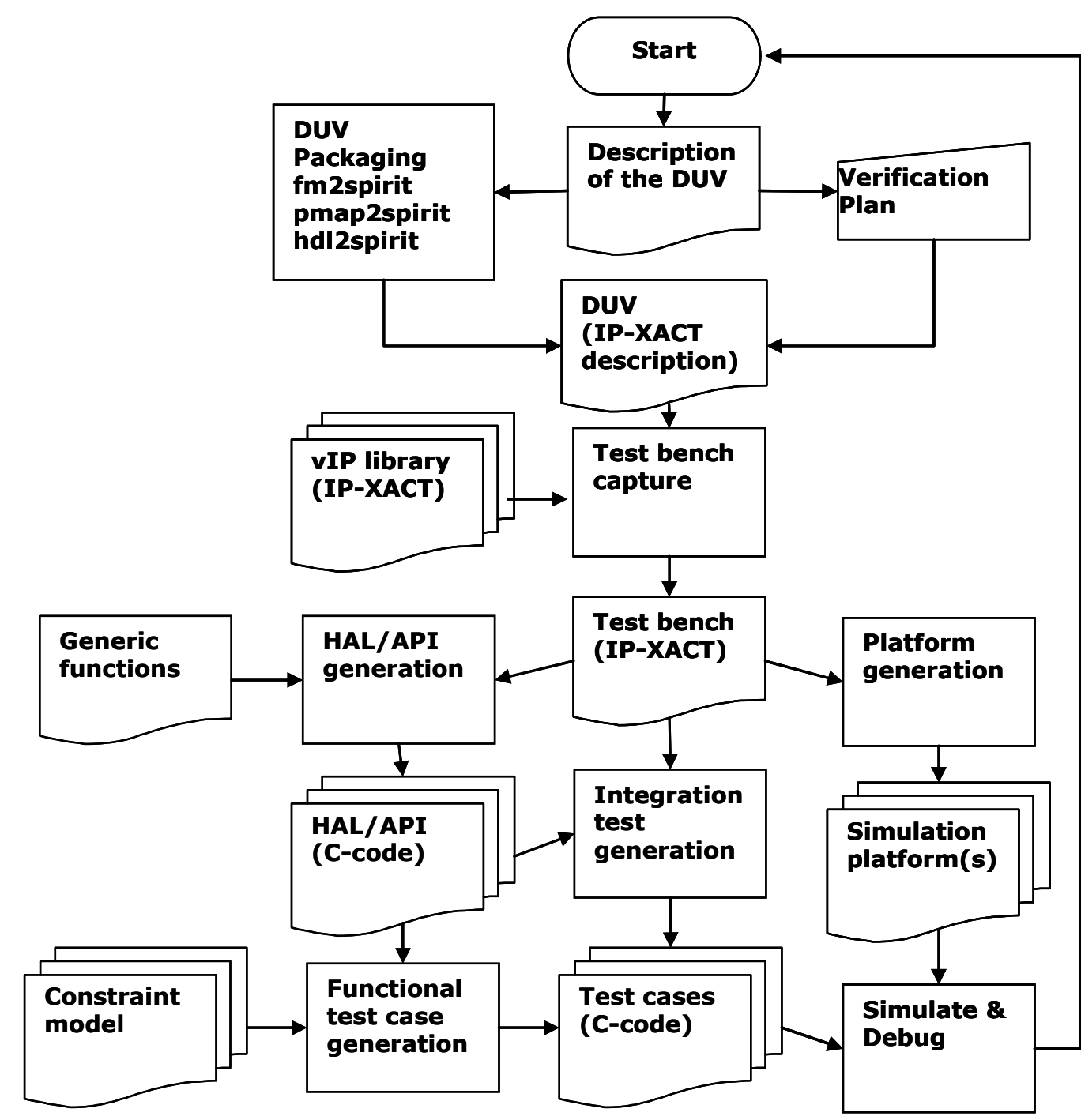

Figure 6 - SAVE flow

In this flow, the verification engineer will receive the Design Under Verification (DUV) source code and its description in document form. Both are needed to generate an IP-XACT description of the DUV, if not already done by the design team, and also to help with DUV understanding and verification plan's writing. The IP-XACT description is automatic generated using the fm2spirit, pmap2spirit and hdl2spirit tools.

The DUV's IP-XACT description is put into a tool that assists capturing (writing) the testbench in a semi-automated way. The description of this testbench is also in IP-XACT format, which allows it be parsed by several back-ends that can be used to generate interconnection test cases. The HAL/API is used in order to facilitate the writing of test cases in $\mathrm{C}$ and the generation of the netlist for the testbench targeted simulation platform in several ways, like RTL and TLM. After compiling the test cases and the testbench, both can be simulated and debugged. 
In the following the steps of the SAVE flow supported by tool are described.

\subsubsection{DUV packaging}

If the DUV's IP-XACT has not been delivered together with the DUV description, it is generated in this step. The description of the DUV is used to generate the IP-XACT description. This description is normally a combination of RTL-code (for identifying the signals and busses) and a (Framemaker)-document describing the registers and address maps.

For verification purposes information needs to be added to the IP-XACT description about the basic functionality of the IP and the usage of each interface and signal. This extra information help in the testbench capture.

For instance: an IP that decodes video will need to be tagged as a streaming component. This type of IP has normally several bus interfaces that need to be tagged with their purpose. For example, a bus interface for accessing the internal registers should be tagged as a control interface, while an interface which accepting the compressed data should be tagged as a streaming interface. Handshake signals for DMA and signals for interrupting requests are also examples that should also be tagged. These tags will allow the testbench capture tool to generate the structures with IP functionality knowledge. For example, if the DUV uses a DMA, the testbench capture tool should automatically insert a DMA-controller into the generated testbench.

These tags were not incorporated to the SAVE yet. Some of them had already defined like streaming, control, DMA and interrupt, but it were not incorporated to the flow. The present state of the DUV packaging step doesn't append these tags in the IP-XACT description.

\subsubsection{Extract verification functions}

The verification plan normally lists all the functional requirements that need to be verified. In a verification environment where the test cases are written in $\mathrm{C}$ and run as firmware, these functional requirements are initiated by $\mathrm{C}$-functions. The definition of these functions should be added to the IP-XACT description of the IP to be tested. These functions should be called by the test cases to perform a specific stimulus into the system, which cover the functional requirement verification.

Checks (checkers) that should and can be implemented using $\mathrm{C}$-functions, e.g. checks that are implemented in firmware, also need to be added to the IP-XACT description of the 
IP. The checkers are responsible to verify that the state of the system after a C-function execution is according to the desirable. A checker can verify if a specific functional requirement or a set of them have success during the verification. In generally we use a pair of C-function definition to a checker, it guarantee the granularity of the test.

This step is not available yet and the Verification Planning Data Study Group from the SPIRIT Consortium is performing a feasibility study.

\subsubsection{Testbench capture}

In this step, the testbench is generated. The design environment uses an external generator which analyzes the tags added to the DUV's IP-XACT description during the packaging process and, based on these tags, plug-in automatically the right verification IP's.

Suppose the example mentioned in the DUV packaging section, in that case the IP's with functionality knowledge to be inserting are a DMA controller, an interrupt trigger, a memory block.

\subsubsection{HAL/API generation}

The design environment will use a tool to analyze the IP-XACT description of the testbench and generate all the getters and setters for all the registers and their fields in the design. The phase consist the focus of this master research. The tool responsible to handle this task was developed in this master. In Section 4 this step is discussed in detail and the tool architecture proposed and implemented are presented.

\subsubsection{Platform generation}

During this step, the IP-XACT description of the testbench is given to the platform generator tool that will generate the netlist for the targeted platform(s). The launchscripts to execute the testbench are also generated.

\subsubsection{Test cases generation}

In the test case generation step the verification engineer uses the generated HAL/API to write directed test cases. The test cases are written following the verification plan. The goal is verify all functional requirements. The HAL/API can also be used by others tools, like Esterel, ISX or InFact in order to generate the test cases automatically. 


\subsubsection{Simulation and debug}

During this phase, one of the platforms previously generated is used to run one or more generated test cases. If the checkers report some errors, the test case is debugged on the given platform. If none test case fail the DUV can be considered as tested.

\subsection{SAVE tools status and work contribution}

The SAVE methodology is being developed gradually. The Table 2 shows the status of the tools and definition documents already available in the SAVE methodology and what will be developed as well this master research contribution.

Table 2 - SAVE tools' status and work contribution

\begin{tabular}{|c|c|c|}
\hline Step & Task & Status \\
\hline \multirow{3}{*}{ DUV packaging } & Register and RTL packaging & Yes \\
\hline & Verification automation tags definition & No \\
\hline & Automation tool & No \\
\hline \multirow{2}{*}{$\begin{array}{l}\text { Extract verification } \\
\quad \text { functions }\end{array}$} & Verification plan packager tool & No \\
\hline & $\begin{array}{l}\text { Write formal test plan model with third party } \\
\text { tool }\end{array}$ & No \\
\hline Testbench capture & $\begin{array}{l}\text { Automatic insertion tool of external testbench } \\
\text { components }\end{array}$ & No \\
\hline \multirow{3}{*}{ HAL/API generation } & HAL/API function header definition & $\begin{array}{l}\text { Work } \\
\text { contribution }\end{array}$ \\
\hline & API-template generator & $\begin{array}{l}\text { Work } \\
\text { contribution }\end{array}$ \\
\hline & HAL generator & $\begin{array}{c}\text { Work } \\
\text { contribution }\end{array}$ \\
\hline Platform generation & Generate platform from IP-XACT description & Yes \\
\hline \multirow{3}{*}{ Test cases generation } & $\begin{array}{l}\text { Automatic generation of functional test cases } \\
\text { using a test plan model }\end{array}$ & No \\
\hline & $\begin{array}{l}\text { Automatic generation of interconnection test } \\
\text { cases }\end{array}$ & No \\
\hline & $\begin{array}{l}\text { Automatic generation of test cases from } \\
\text { formal test plan model and generated API } \\
\text { functions }\end{array}$ & No \\
\hline Simulation and debug & Simulate and debug test cases on platforms & Yes \\
\hline
\end{tabular}

As showed in Table 2 nowadays the tools and documents foreseen by SAVE are partially implemented and defined. The process to generate the IP-XACT description of 
the SoC in automatically manner is ready (Register and RTL packaging). The Platform Generation and the Simulation and Debug environment also can be generated in an automatically manner supported by an IP-XACT Design Environment. Analyzing the SAVE flow was detected that before start the next tools development firstly was needed guarantee that an IP-XACT description of a SoC offers enough information to create a tool able to generate a standard interface that provide a high level abstraction to access the SoC components. This premise aims guarantee that a Hardware Abstract Layer (HAL) could be automatically generated by a tool just using the information provided in the IP-XACT description.

This work improves the SAVE methodology by investigating and proposing an implementation model for the HAL/API generation tool. The HAL/API objective is to assist the verification engineer with the test case coding. With that API the verification engineers can focus their work on functionality tests, once the infrastructure to manipulate the basics elements, registers and their fields, is implemented by the HAL generator tool.

The proposed approach consists in three steps: HAL/API function header definition, API-template generator and HAL functions generator. In the first step was defined the getters, setters and resets functions declarations. After that a template for the API functions was defined concluding with the HAL generator implementation.

\subsection{Final Considerations}

This Section presented the Spirit Assisted Verification Environment (SAVE) methodology. Basics concepts related SAVE and its flow were discussed. At the end of the Section the SAVE development status was presented as well this master research contribution pointed.

The next Section detail the HAL generator functionalities and the architecture proposed for implementation in this master research. 


\section{The Proposed Hardware Abstract Layer (HAL)}

\section{Generator}

The HAL provides all calls that are needed to read or write registers and their fields. The output of the code generator consists of a set of functions in the $\mathrm{C}$ language that allows the access of the registers contents in a $\mathrm{SoC}$ in a high level of abstraction. The verification engineers can manipulate the registers values just by calling those functions in their $\mathrm{C}$ test cases codes.

\subsection{How the HAL generator works}

The HAL generator procedures a set of steps until generate the API functions. In Figure 7 the step flow is shown. The first step captures the IP-XACT description of the testbench. The capture is done by using the Tight Generator Interface (TGI) methods, which allow analyzing each IP contents (bus interfaces, memories, address blocks, registers, fields, etc). As the capture going on a SAVE representation of the IP-XACT description is generated using objects specially defined to represent the IP-XACT elements using a property representation defined in this master research. These objects are detailed in Section 4.3. This is the SAVE manner to represent the testbench.

Once the IP-XACT is captured and the SAVE representation of the testbench is ready, starts the step that search for each slave interface present in the testbench. The slave's interfaces are the entry point to mount the registers and their fields address. In this step all slaves' interfaces are labeled with the base address regarding the main processor master interface. This is a complex step because all busses hierarchy and connections need to be checked. In this step a depth-first search is procedure through the busses hierarchy until all slaves' interfaces were found. The final stage is the functions generation. A search engine looks for registers and fields in the SAVE's IP-XACT representation generating the HAL functions. 


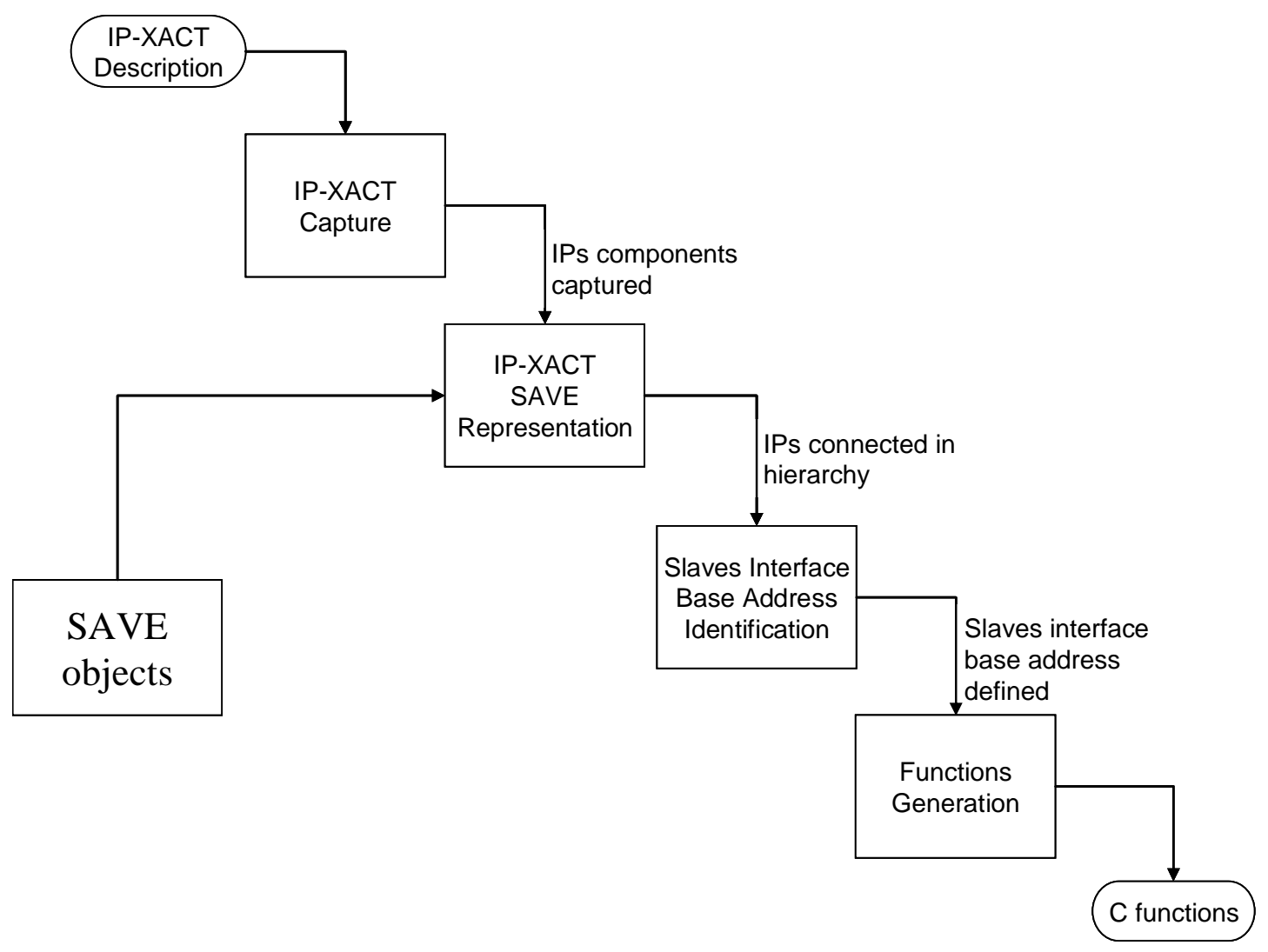

Figure 7 - HAL generator flow

\subsection{Registers and fields IP-XACT representation}

IP-XACT defines a set of elements to describe a register, some of them are mandatory, and others are optional. Each register is labeled by a name and a description. The other nine elements used to describe a register are shown below:

1. $\operatorname{dim}$ (optional): assigns dimension to the register;

2. addressOffset (mandatory): describes the offset of the register inside the address block that contains it;

3. size (mandatory): is the width of the register, measured in bits;

4. volatile (optional): when true indicates in the case of a write followed by read, or in the case of two consecutive reads, there is no guarantee what is returned by the read on the second transaction or that this return value is consistent with the write or read of the first transaction. The attribute implies there is some additional mechanism by which these registers can acquire new values other than reads/writes/resets and other access methods known to IP-XACT. 
5. access (optional): indicates the accessibility of the register. If this is not present, the access is inherited from the containing addressBlock. There are three choices: 1) read-write: Both read and write transactions may have effect on this register. Write transactions may affect the contents of the register and read transactions return a value related to the values in the register. 2) read-only: A read transaction to this address returns a value related to the values in the register. A write transaction to this register has undefined results. 3) write-only: A write transaction to this address affects the contents of the register. A read transaction to this register has undefined results. Depending on this element's value, the HAL will generate get, set or get/set for the register;

6. reset (optional): indicates the value of the register contents when the device is reset;

7. field (optional): describes any bit-fields in a register. Details about it are given below;

8. parameters (optional): describes any parameter names and types when the register width can be parameterized;

9. vendorExtensions (optional): adds any extra vendor-specific data related to this register;

The Figure 8 in following shows an IP-XACT description code example for a register element.

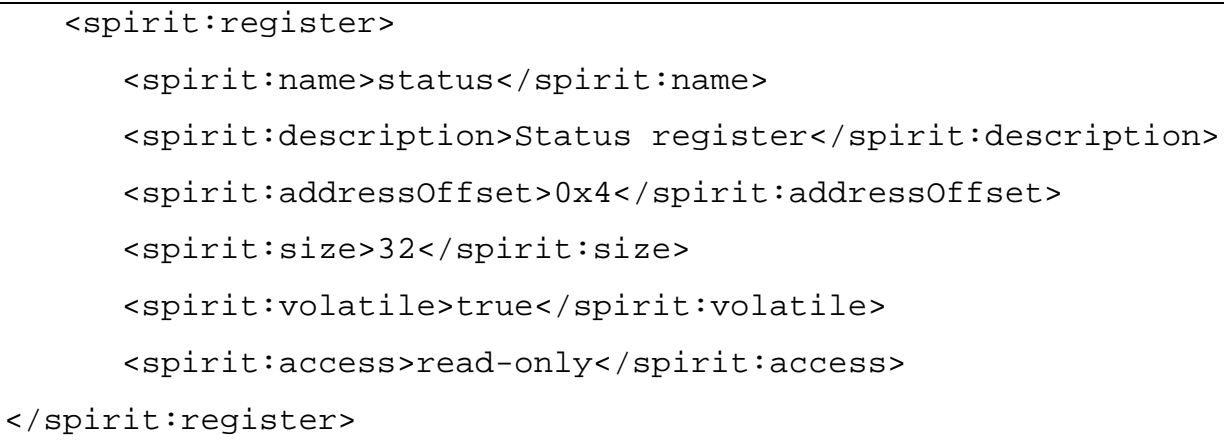

Figure 8 - IP-XACT description for a register element

The field element contained inside a register also has a set of elements following the same idea showed before. Besides a name and description, the IP-XACT also defines:

1. bitOffset (mandatory): describes the offset (from bit 0 of the register) where this bit-field starts;

2. bitWidth (mandatory): is the width of the field, measured in bits; 
3. access (optional): same description of the register element;

4. values (optional): lists the set of legal values that may be written to the bitfield. Each value is composed of a value, a description and a name;

5. parameters (optional): details any additional parameters that describe the field for generator usage;

6. vendorExtensions (optional): same description of the register element.

The Figure 9 in following shows an IP-XACT description code example for a field element.

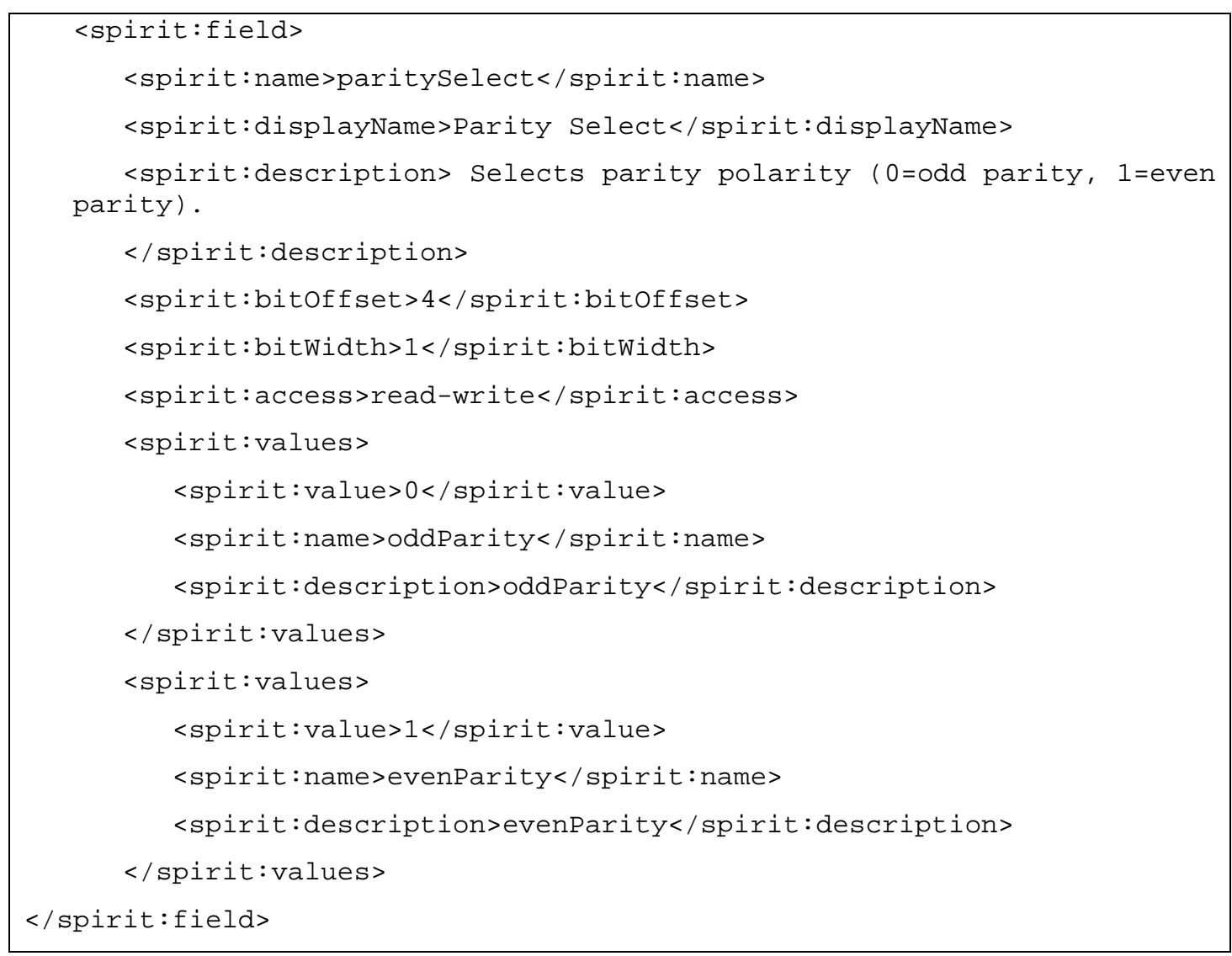

Figure 9 - IP-XACT description for a field element

The XML information shown above is accessed by the HAL generator through the TGI API methods. Due to the IP-XACT standard defines just the TGI API methods interface without providing their implementation; we needed a tool that provides the methods implementation. In this work the tool Magillem Design Environment [17] was used.

The functions generated by the HAL should be capable to access any register in the SoC hierarchy. This means that to generating these functions the HAL generator needs to define the register address regarding the processor master interface. Each register (box 1 in Figure 10) is part of an address block (box 2 in Figure 10), which in turn is part of a 
memory map (box 3 in Figure 10). Each memory map is accessed through a slave interface (box 4 in Figure 10) connected to the system bus hierarchy. Figure 10 represents this relation; in the example the address of such register is defined by the sum of all addresses, in this case $0 \times 1114$.

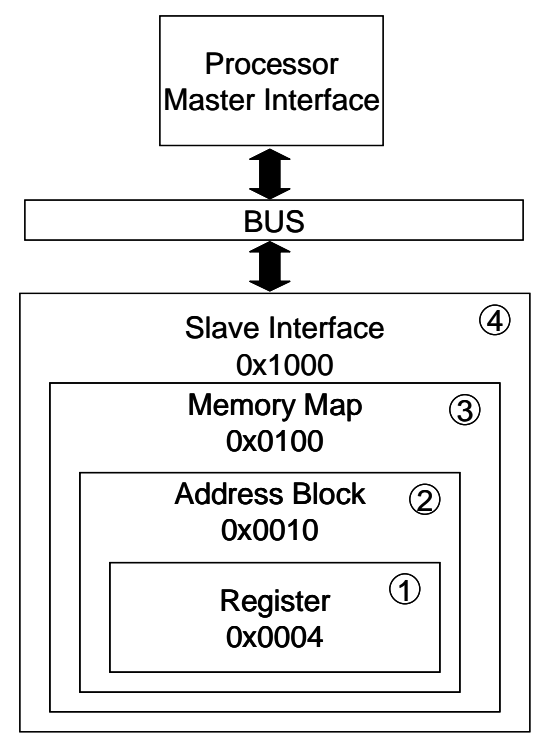

Figure 10 - Addressing Registers

Each slave interface's base address is defined regarding the master interface (base address zero), which is connected with the CPU (processor) in charge of running the test cases during the SoC functional verification. To define the registers' address the HAL generator starts analyzing the IP-XACT description from the top model and goes into the SoC hierarchy until to reach all components. For each bus channel identified, sub-systems can be found, so when a sub-system is identified a recursive process starts to analyze each IP in that sub-system. In Appendix B the algorithm used to determine the slaves interfaces base address is stated in a flow chart.

Figure 11 shows a SoC example with a processor, busses, channels, subsystems, among others. This SoC is compounded of following IP blocks:

- Processor: CPU in charge of running the system application. It is a Leon2 processor, a 32-bit microprocessor core based on the SPARC-V8 RISC architecture and instruction set;

- Leon2Ahbbus: AMBA AHB bus [1] to connect the processor cache controllers to the memory controller and other high-speed blocks units. In this example the processor is the only master on the bus, while two slaves are provided: the RAM module and the APB subsystem; 
- AhbRam: a RAM module to providing high-speed on-chip memory storage capacity;

- Cgu: a clock generator unit used to handles the clock of the system;

- Rgu: a reset generator unit used to handles the reset of the system;

- ApbSubSystem: a subsystem contents an AMBA APB bus, a timer, an interrupt controller, twos serial interface and APB bridge.

○ APBbus: a peripheral bus for low-speed on-chip communication;

- Timer: a timer block used to control the sequence of an event or process;

- Uart: a universal asynchronous receiver/transmitter block to external serial communication;

o Interrupt: an interrupt controller block in charge to handles the system internal and external interrupts.

- APB bridge: this block implements a bridge between the APB and the AHB busses. 


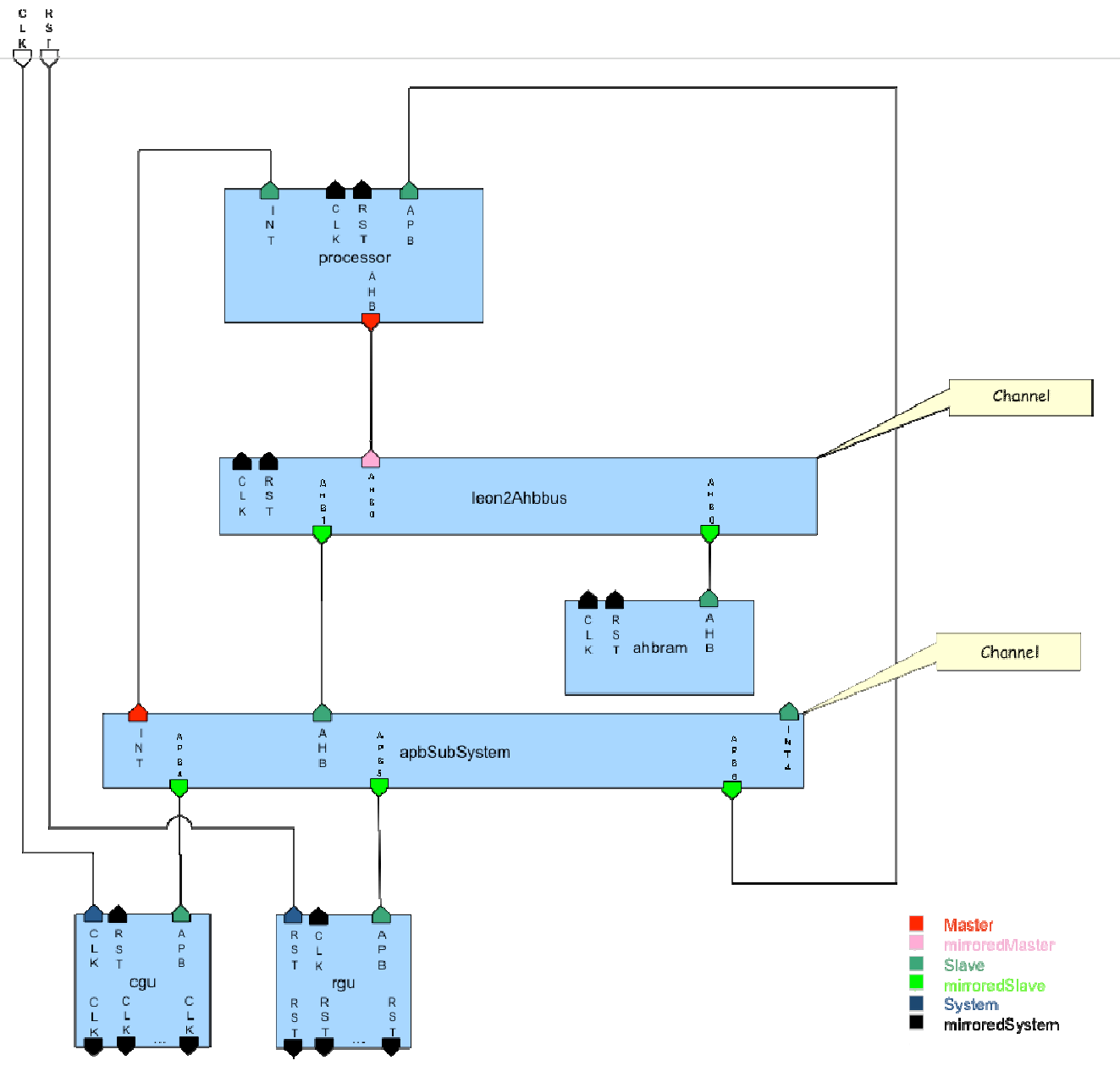

Figure 11 - SoC example

The HAL generator analyzes each IP block (processor, leon2Dma, leon2Ahbbus, ahbram, cgu, rgu) and Sub-Systems (apbSubSystem) IP-XACT description, capturing its elements. Once all elements are captured and the SoC hierarchy is known, the HAL generator search for channels (leon2Ahbbus) and bridges (ApbSubSystem) through the hierarchy calculating the base address of each slave interface. At the end of this process a new representation of the SoC is generated. Finally, the code generation process starts working on this representation to create the $\mathrm{C}$ functions.

\subsection{The HAL generator architecture}

The HAL generator was developed in Java and is composed of five Java packages. The decision to use this programming language was due to the fact that the TGI API methods are specified in Java and the Design Environment [17] is based on a Java platform (Eclipse IDE [5]). The TGI API methods are the standard manner defined by The Spirit Consortium [21] to access the IPs and designs information through their IP-XACT description. The 
implementations of these methods are given by the Design Environment (DE). In SAVE methodology the DE selected was Magillen Design Environment [17].

Five Java packages were defined: Target, SAVE, SAVE_SPIRIT, Adapter, TGI_IPXACT. The five packages and their relations are shown in Figure 12. The package Target contents the main method, which is in charge of initialize the system. It is responsible for starting the TGI interface and the Adapter.

The package Adapter captures the IP-XACT description using the TGI methods. For each element captured there is a Java basic class or an attribute for representing it. These classes and attributes are found in the SAVE_SPIRIT package.

When the Adapter finishes the capturing process a representation of the IP-XACT description for the testbench was built using the classes from the SAVE_SPIRIT. For this representation we called SAVE IP-XACT representation.

The SAVE package gets the SAVE IP-XACT representation and works with that structure looking for registers. As the registers are found the HAL functions are generated. Each package is detailed in sequence.

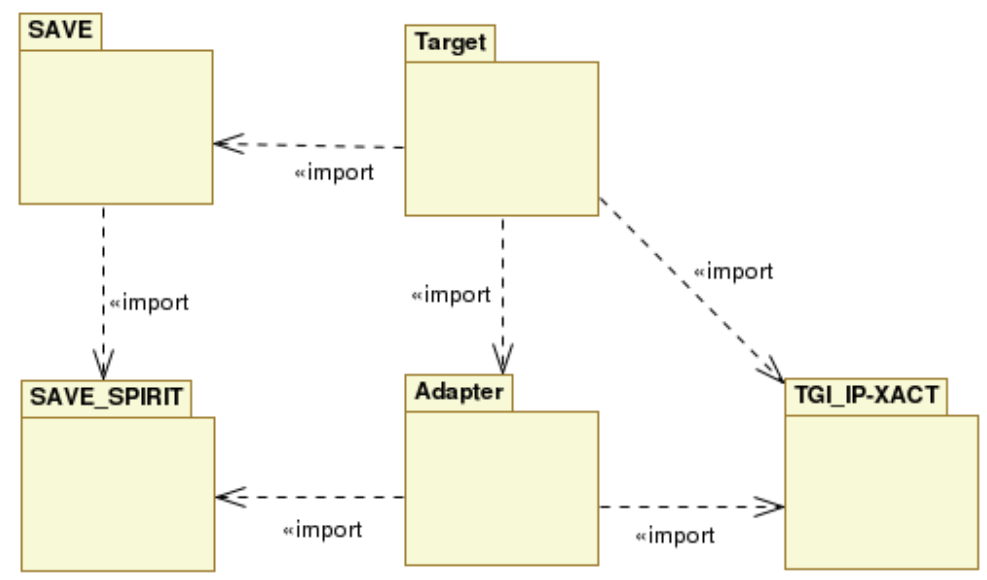

Figure 12 - Packages relations

\subsubsection{TGI_IP-XACT package}

The TGI_IP-XACT package implements the TGI's methods. The implementation of this package is given by Magillem Design Environment [17] tool. The packages that need to use the TGI's methods should import it.

\subsubsection{SAVE_SPIRIT package}

The SAVE_SPIRIT package is composed of a set of classes that represent the IPXACT objects and their elements. These Java objects are used to build a SoC 
representation easier to be manipulated - this is the SAVE manner for representing an IPXACT description of a SoC. In the Adapter package's explanation more details about the SAVE' IP-XACT representation are given. Figure 13 shows the UML classes diagram for this package. A brief description of each class role is given below. 


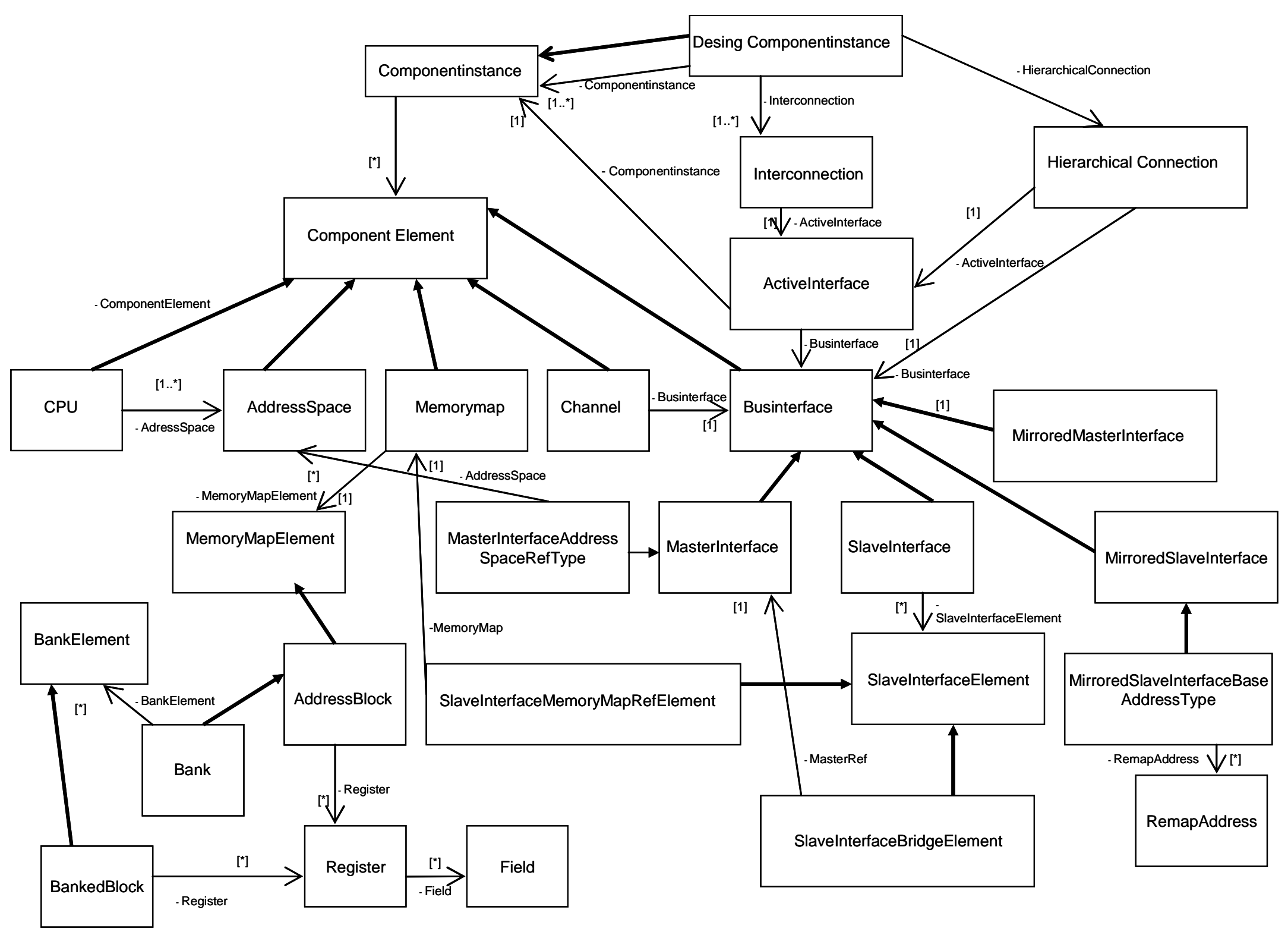

Figure 13 - SAVE_SPIRIT class diagram 
ComponentInstance: represents an IP instantiated in a SoC. The object ComponentInstance has a list of all elements and interconnections found in an IP.

DesignComponentInstance: represents a hierarchical model. In a SoC description we can find many components or designs. This object stores a list of ComponentInstance which composes the Design. In that list we can also find other DesignComponentInstance as this class extends the ComponentInstance. This relation represents a sub-system when instantiated by a SoC. In Error! Reference source not found. the apbSubSystem and the top model are instantiated as DesignComponentInstance, while all other models are instantiated as ComponentInstance. To represent the relations between those instances, interconnections and hierarchical connections are instantiated by the DesignComponentInstance object.

ComponentElement: represents an abstraction of the component elements found in IP-XACT description. This work focuses on implementing the elements classes' representation necessary to create the HAL functions. The IP-XACT defines other elements not implemented here but those can be done in an easy way due to the extensible architecture proposed.

Interconnection: represents the connection between two ComponentInstances. Each interconnection has a pair of ActiveInterface.

HierarchicalConnection: represents a connection between a sub-system and a higher level model in the hierarchy. The sub-system uses a bus interface to make the connection between an IP inside its architecture and a higher model in the hierarchy. For instance, in Error! Reference source not found. an interrupt controller (INT, master interface) model that belongs to the apbSubSystem has a hierarchical connection with the processor (INT, slave interface).

ActiveInterface: to identify a pair of bus interface and component, IP-XACT defines the active interfaces. The active interfaces are addressed by the interconnection and hierarchical connections to represent the bind between IPs.

CPU: is a ComponentElement and identifies that an IP has a processor unit. A CPU element has an address space which defines the range of addresses which the master interface can access. 
AddressSpace: this class extends ComponentElement and is defined as a logical addressable space of memory. Each master interface can be assigned a logical address space.

MemoryMap: this class extends ComponentElement and can be defined for each slave interface of a component. A MemoryMap contains a list of MemoryMapElement; these elements are accessed through the slave interface that defines it.

MemoryMapElement: represents an abstraction of the memory maps elements defined in IP-XACT. Each memory map element contains a base address. In this work Address Block and Bank elements were implemented. SubSpaceMap element was not implemented once it doesn't give relevant information to HAL functions generation.

Channel: this class extends ComponentElement and represents a collection of connections between multiple internal bus interfaces. A channel has a list of bus interfaces.

BusInterface: this class extends ComponentElement and represents an abstraction of the bus interfaces defined in IP-XACT. In this work four bus interfaces types were implemented: master interface, slave interface, mirrored master interface and mirrored slave interface. The other bus interfaces defined in IP-XACT were not implemented.

MasterInterface: extends BusInterface and initiates transactions. The master element can be associated to an address space with which this master interface can generate transactions.

MasterInterfaceAddressSpaceRefType: extends MasterInterface and represents a master interface with an association with an address space element.

SlaveInterface: this class extends BusInterface and responds to transactions. The slave interface can reference a memory map element and can also be used in a bridge application to "bridge" a transaction from a slave interface to a master interface. The SlaveInterfaceElement implements these two behaviors of a slave interface, each slave interface has a list of these elements.

SlaveInterfaceElement: represents an abstraction of both applications implemented by a slave interface. The SlaveInterfaceMemoryMapRefElement and SlaveInterfaceBridgeElement classes implement these applications.

SlaveInterfaceMemoryMapRefElement: this class extends SlaveInterfaceElement and stores the reference to the memory map element accessed by this interface. 
SlaveInterfaceBridgeElement: this class extends SlaveInterfaceElement and stores the reference to the master interface to which the transactions must be forwarded.

MirroredMasterInterface: this class extends BusInterface and implements the mirrored master interface defined in IP-XACT. As the name suggests, a mirrored interface has the same (or similar) ports as its related direct bus interface, but each port direction or initiative is reversed.

MirroredSlaveInterface: this class extends BusInterface and implements the mirrored slave interface defined in IP-XACT. A mirrored slave interface may contain additional baseAddress information, which can redefine the original interface address. The MirroredSlaveInterfaceBaseAddressType class implements this extension.

MirroredSlaveInterfaceBaseAddressType: this class extends MirroredSlaveInterface and implements a mirrored slave interface with additional baseAddress information. These additional addresses redefine the slave interface address and range connected with this mirrored interface. Each additional baseAddress can be associated with a state, which determines if the redefinition occurs or not. The pair baseAddress and state are represented by the class RemapAddress. MirroredSlaveInterfaceBaseAddressType class has a list of RemapAddress.

RemapAddress: represents a pair of baseAddress and state instantiated by the MirroredSlaveInterfaceBaseAddressType class.

AddressBlock: this class extends MemoryMapElement and describes a single, contiguous block of memory that is part of a memory map. An address block contains the attributes range and width, which define its limits. A set of registers can appear inside the address block.

Bank: this class extends MemoryMapElement and allows multiple address blocks, banks or subspaceMap that can be concatenated together horizontally or vertically as a single bank. Besides the base address and alignment attributes a Bank object may have a list of other bank elements, which can be a bank block, a subspace or another bank. In this work just the bank block element was implemented, due to constraints in the Design Environment.

BankElement: this class represents an abstraction of the bank elements. Although just the bank block element was implemented in this work, the support for the other bank elements is present to allow an easy implementation in the future. 
BankedBlock: this class has the same role of the AddressBlock class, however, it does not define a new base address.

Register: this class represents the IP-XACT description for a register. Details about register attributes are shown in the sub-section How the HAL generator works.

Field: this class represents the IP-XACT description for a bit-field defined in a register. Details about field attributes are shown in the sub-section How the HAL generator works.

\subsubsection{Adapter package}

The package Adapter is responsible for generating the SAVE representation of an IPXACT description. It is implemented by the class with the same name and imports the SAVE_SPIRIT and TGI_IP-XACT packages. Once the Design Environment starts the HAL generator execution, the Adapter parses all the IP-XACT description using TGI's methods calls and creates the instances and elements representation for the SoC description using the classes from the package SAVE_SPIRIT.

The Adapter creates an abstraction level of the TGI's methods for the package SAVE. The insertion of this extra layer is due to the fact that almost all TGI's methods return arrays of strings. This information is difficult to be manipulated and does not offer significant information if we see it as a single method access. With the object-oriented representation of the IP-XACT, each Java Object provides all information regarding the element or the component that it represents and also the meaning of the object instance.

This extra layer also allows the separation of system's functionalities. While the Adapter is in charge of capturing the IP-XACT description and creating the SAVE's IP$\mathrm{XACT}$ representation, the SAVE package is responsible for generating the $\mathrm{C}$ code.

Finally that decision guarantees maintainability for the system as the TGI access is separated from the system functionality and possible changes on TGI definition do not affect the code generation tool, as the SAVE's IP-XACT representation is respected.

The IP-XACT description's capture is done in three steps. Figure 14 shows the steps flow. Firstly, the component instances are identified and a hierarchy tree of the SoC components is created. The graph showed in step 1 represents the SoC hierarchy. The vertices in red represent a design with others components on its hierarchy. Once the hierarchy tree is known, each component instance has its elements (CPU, AddressSpace, MemoryMap, Channel, BusInterface, etc) identified. Each vertex is examined and its 
components identified. In the third step the slave interfaces have their base address calculated. A depth-first search algorithm looks for each slave interface, once a slave interface is found during the backtrack process the slave interface has its base address defined in the SoC hierarchy. This address is used to define the base address of the component instance in the SoC hierarchy. This address also is used to define the base address of the registers like mentioned in Section 4.2 in Figure 10.

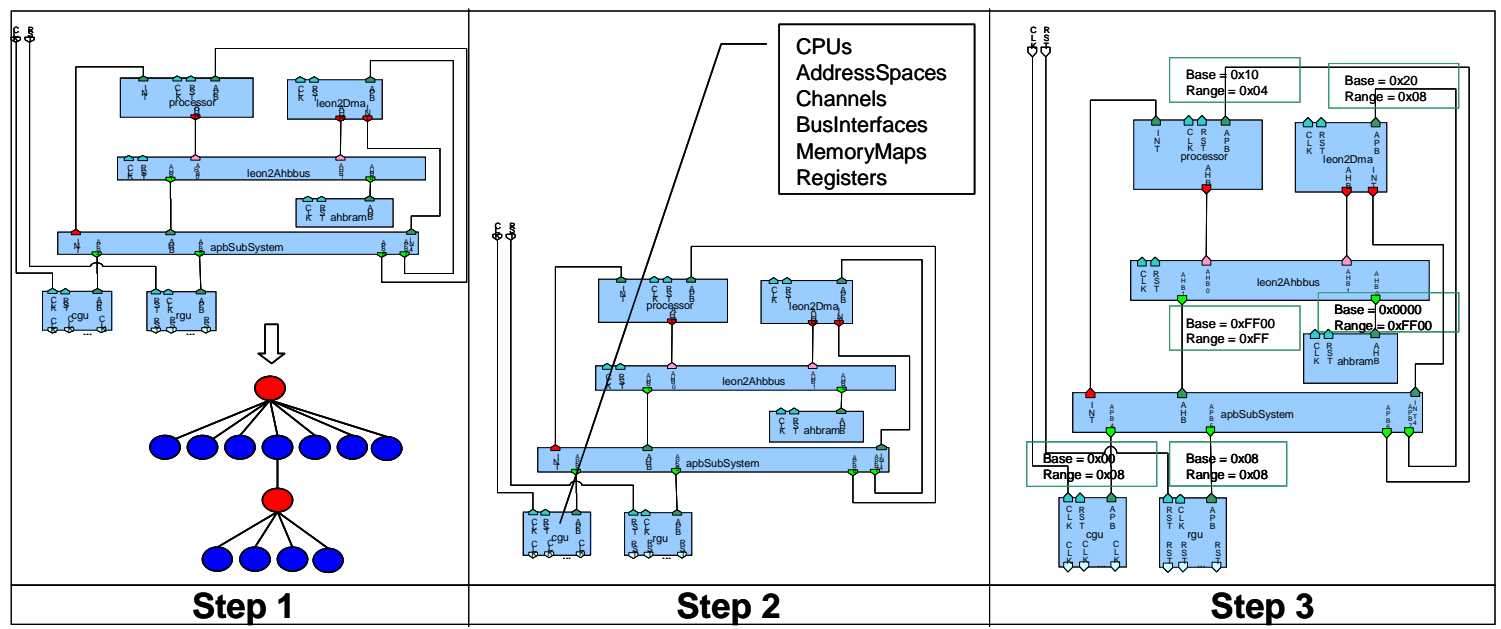

Figure 14 - IP-XACT description capturing steps

\subsubsection{SAVE package}

Once the Adapter package has captured the IP-XACT description and created the SAVE's IP-XACT representation, the $\mathrm{C}$ code generation process starts. This package is implemented by the class called SAVE.

The first step of this process is to create a file with all addresses components, registers and fields address. In this file the IPs base address and the registers as well as their fields' offsets are defined.

The IPs base address is used by the verification engineer to support the test cases coding. The registers and fields offsets are used by the gets and sets functions. They are also useful to gives an entire address map view of the SoC. This file can be compared with Framemaker document - a document used at the beginning of the functional verification that defines the registers' address map - to validate the code generated.

Figure 15 shows part of the code generated for the SoC showed in Error! Reference source not found.. The C define called "BASE_ADDRESS" represents the processor master interface address. In sequence we have the IPs base address regarding the master interface processor; this value is used as an argument for the HAL functions calls. The 
following $\mathrm{C}$ defines represent the registers and their fields offset into the IP. In that example we can see just the offset for the interrupt mask and priority level register. This register belongs to the interrupt control IP.

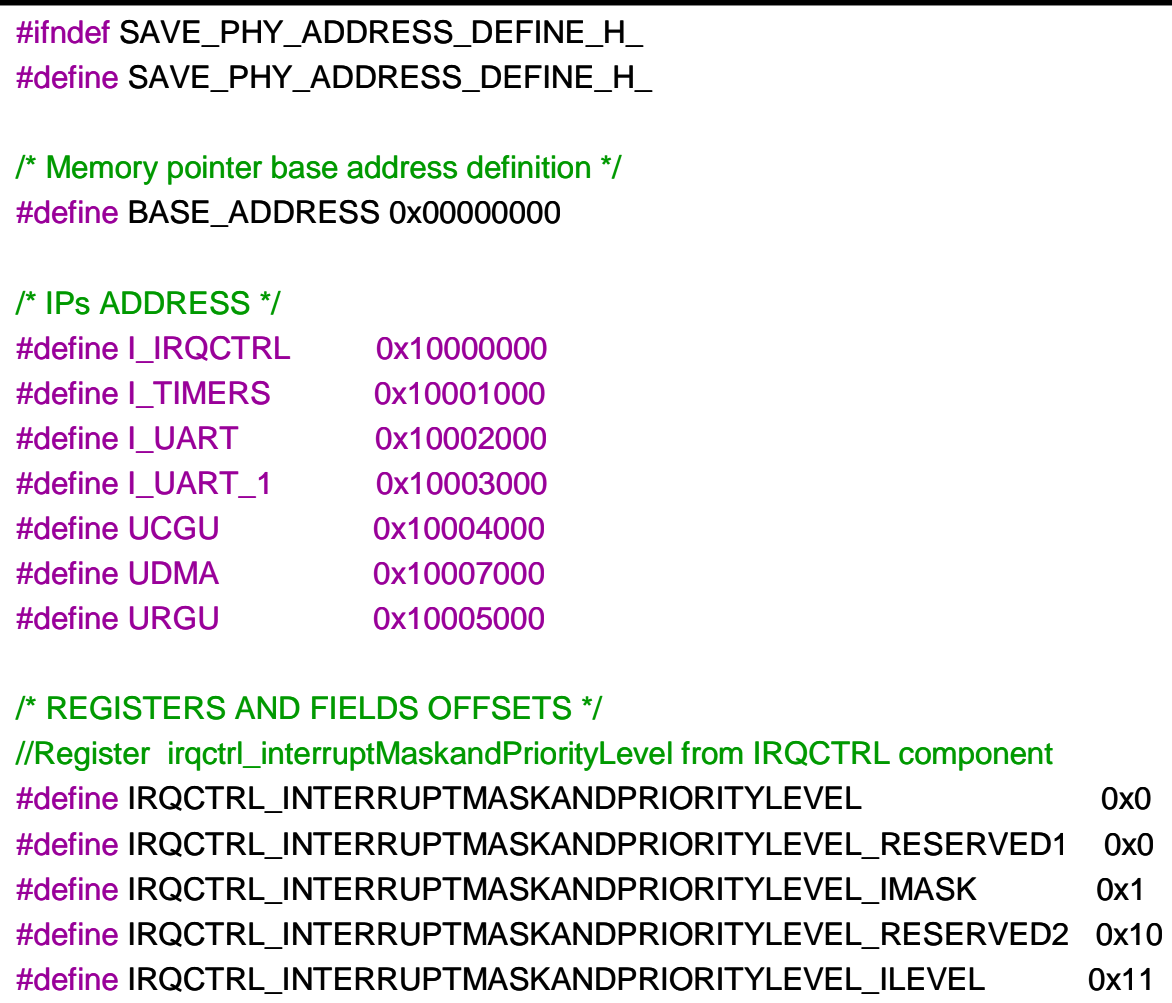

Figure 15 - Defines file content

The second part of the code generation process is the actual functions' generation. For each register and field getters and setters methods are generated. If a reset value is defined in the IP-XACT description and the register is writable the reset method is also generated.

A standard nomenclature for the functions naming was defined. There are four fields. The first field identifies if the call is a write to (set), a read from (get) or a reset of (reset) the register. The second field identifies the component name it means what instance should be manipulated. The third field defines the name of the register into the IP, which should be accessed. Finally the last one refers to the field intend to be addressed.

\$ $\{$ set $\mid$ get $\mid$ reset $\}$ \$ $\{$ component_name\}_\$ $\{$ register_name $\}$ \$ $\{$ field_name $\}$

The registers can be read and written in two manners. The first one allows read and write value in the entire register. In the second manner the access is made by field, so just the specific field value is read or written. Case a register doesn't content fields the unique way to manipulate it is reading or writing the entire value. 
Get and reset calls only take one argument. This argument indicates the base address of the IP being addressed. Set calls take two arguments; the first is the base address of the IP and the second the value to be written.

Two C files are generated in this step, one with the functions declarations and another with their definitions. Figure 16 shows part of a declaration file for the same SoC. 
$I^{*}$ Include definition files */

\#ifndef SAVE_FUNCTIONS_H_

\#include "SAVE_functions.h"

\#endif

ifndef SAVE_PHY_ADDRESS_DEFINE_H_

\#include "SAVE_phy_address_define.h"

\#endif

$/{ }^{*}$ Memory pointer declaration */

volatile unsigned int * ptr_memory;

* Function: get_irqctrl_interruptMaskandPriorityLevel

* Register Description: Register responsible to configure the Interrupt Mask and Priority.

* This function returns the irqctrl_interruptMaskandPriorityLevel register value.

* instance_address: the IP base address

* return: the register value

*/

long int get_irqctrl_interruptMaskandPriorityLevel(unsigned int instance_address) \{

ptr_memory $=($ volatile unsigned int * $)$

(BASE_ADDRESS+instance_address+IRQCTRL_INTERRUPTMASKANDPRIORITYLEVEL); return *ptr_memory;

$/ *$

* Function: reset_irqctrl_interruptMaskandPriorityLevel

${ }^{*}$ Reset value: 0x0. Mask value: 0xfffe

* This function performs the irqctrl_interruptMaskandPriorityLevel register reset operation.

* instance_address: the IP base address

*/

long int reset_irqctrl_interruptMaskandPriorityLevel(unsigned int instance_address) \{

ptr_memory $=\left(\right.$ volatile unsigned int $\left.{ }^{*}\right)$

(BASE_ADDRESS+instance_address+IRQCTRL_INTERRUPTMASKANDPRIORITYLEVEL); ${ }^{*}$ ptr_memory $=$ (get_irqctrl_interruptMaskandPriorityLevel(instance_address) \& 0xfffe);

*ptr_memory $=0 \times 0$;

* Function: set_irqctrl_interruptMaskandPriorityLevel

* Register Description: Register responsible to configure the Interrupt Mask and Priority.

* This function sets the irqctrl_interruptMaskandPriorityLevel register value to new_value.

* instance_address: the IP base address

* new_value: the new value to set

*/

long int set_irqctrl_interruptMaskandPriorityLevel(unsigned int instance_address, long int new_value) \{ ptr_memory $=($ volatile unsigned int * $)$

(BASE_ADDRESS+instance_address+IRQCTRL_INTERRUPTMASKANDPRIORITYLEVEL); *ptr_memory = new_value;

\}

Figure 16 - Declaration functions file content 
The variable ptr_memory is a pointer that is updated in every function call. Its initial value by default is zero. An offset is applied using the instance_address parameter, the register's base address and the field's offset in case of a field access. This variable is declared with the modifier volatile to force the compiler to generate load and store operations in every variable access. In each function comments the register or field description is shown. In case of a reset function, the reset value and mask are shown.

At the end of the code generation process, three $\mathrm{C}$ files are generated: SAVE_phy_address_define.h (base address and offset defines), SAVE_functions.h (declarations) and SAVE_functions.c (definitions). By default these files are created at "/tmp/" directory. To change it the SAVE.java class can be modified.

\subsubsection{Target package}

The package Target content the function main, which is responsible for initializing the HAL generator. A class with similar package names instantiates the Adapter and SAVE classes. When the Design Environment calls the generator, the Target class initializes a TGI object and gets the Design ID through the TGI's method. After that the Adapter is initialized and the capture of the IP-XACT description starts. Once the SAVE's IP-XACT representation is created, the SAVE class is instantiated and the functions files are created. 


\subsection{The HAL generator coding effort}

The aiming of this Section is given to the reader the general idea of the coding effort to implement the HAL generator. The coding phase took three months of half man power. The coding phase considering Java coding, unit tests coding, fixing bugs and rework due to architecture updates.

The Table 3 below shows the code lines numbers for each package. The TGI_IPXACT package implementation was given by a third party tool [17]. A logical code line means any code line with more than 3 characters that are not between comments.

Table 3 - HAL generator code line numbers

\begin{tabular}{|l|c|c|c|}
\hline Packages & Classes & $\begin{array}{c}\text { Logical Code } \\
\text { Lines }\end{array}$ & $\begin{array}{c}\text { Lines of Code w/ } \\
\text { Comments }\end{array}$ \\
\hline Adapter & 1 & 1485 & 1813 \\
\hline SAVE & 1 & 594 & 739 \\
\hline SAVE_SPIRIT & 41 & 1512 & 2180 \\
\hline TARGET & 1 & 29 & NA* \\
\hline TGI_IP-XACT & NA* & NA* & 4774 \\
\hline TOTAL & 44 & 3620 & \\
\hline
\end{tabular}

*NA means "not applicable" once this package implementation was given by a third party tool. The total line coding considered is zero.

\subsection{Final Considerations}

This Section presented the Hardware Abstract Layer generator tool proposed and implemented in this master research. The tool flow is compound of four steps: IP-XACT capture, IP-XACT SAVE representation, slaves interface base address identification and functions generation.

The elements used by IP-XACT to represent the registers and their fields were introduced as well how they are pointed in the SoC hierarchy. The tool architecture was presented and its five packages detailed. The TGI API implementation from Magillen 
Design Environment [17] was used to manage the access to the SoC IP-XACT description. Finally the effort regarding the Java coding of the tool was presented.

The next Section describes a case study illustrating the use of the HAL generator tool in a SoC functional verification context. 


\section{Case Study - Using the HAL Generator in a SoC Functional Verification}

This Section presents a case study of the HAL generator tool in a SoC functional verification context. A SoC platform delivered by Gaisler Research Group based on Leon processor was used as case study.

\subsection{SoC Description: Leon2 platform}

In this case study a RTL Leon2 platform from Gaisler Research Group [8] was used. The initial idea was to use the platform released in IP-XACT 1.4 version, which is also based on the Leon 2 processor, but the platform was not completely ported in the IP-XACT 1.4 release forcing the migration to the original platform provided by Gaisler Research Group and build a customized IP-XACT description for it. The main problem with the platform delivered in IP-XACT 1.4 release consists that the Leon2 processor was replaced by a bus functional model instead of to use a real CPU block. Its means that CPU binary codes generated by the Leon compiler could not be load in the platform therefore the test cases generated in $\mathrm{C}$ and compiled could not run in the platform. A bus functional model just enable read and write routines, it is not capable to decode instructions like a CPU.

An overview of the platform from Gaisler Research Group can be seen in Figure 17. This platform is the same one presented in Section 4.2. In this block diagram the rectangle represents the apbSubSystem. 


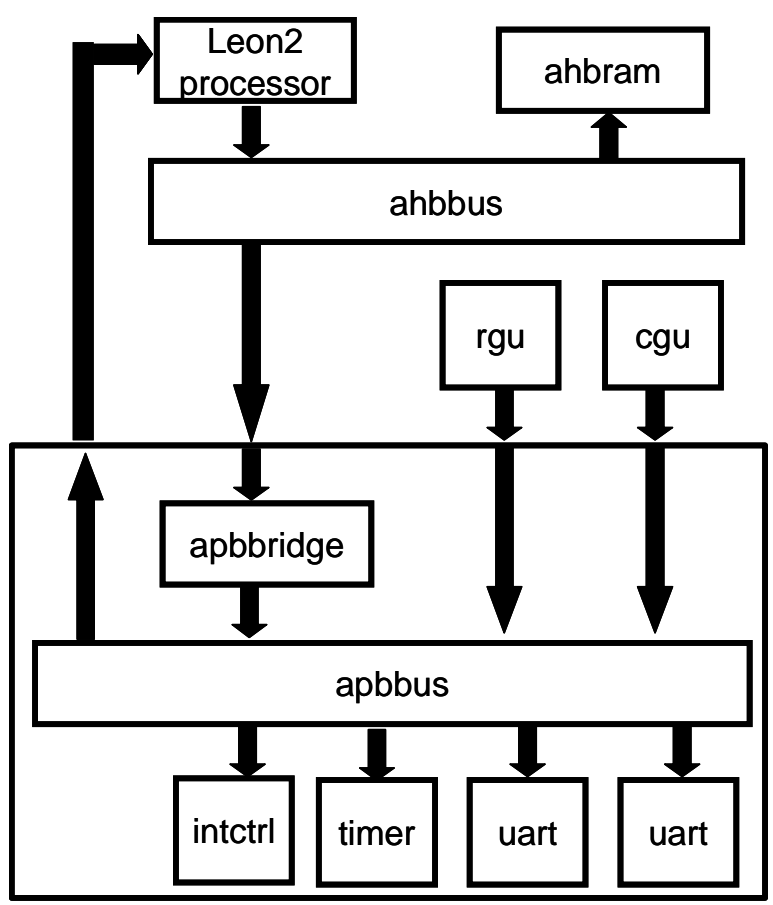

Figure 17 - Platform block diagram

In the platform, the Leon2 is in charge of executing the $\mathrm{C}$ test cases. The tests cases source codes were compiled using the sparc-elf-gcc cross-compiler. The AHB and APB AMBA [1] buses are instantiated and the apbbridge module in the apbSubSystem is in charge of to connect them. The ahhram box represents the instruction and data ram as well as the memory controller. The uarts pins were connected in a cross-strap way to allow data exchange between the both uarts.

\subsection{Universal Asynchronous Receiver/Transmitter (UART) module}

The functionalities of the Universal Asynchronous Receiver/Transmitter (UART) IPs were chosen to be verified in this case study. The functional verification of the all SoC functionalities would take long time and will be a future work.

UART is the key component of the serial communications subsystem of a computer system. The UART takes bytes of data and transmits the individual bits in a sequential fashion. At the destination, a second UART re-assembles the bits into complete bytes. A UART is used to convert the transmitted information between its sequential and parallel form at each end of the link.

The functional verification intends guarantee that the module works as its specification document. The UART IP used in this case study according the specification provide by Gaisler Research Group [8] support data frames with 8 data bits, one optional parity bit 
and one stop bit. Hardware flow-control is supported through the RTSN/CTSN hand-shake signals. Figure 18 shows a block diagram of a UART.

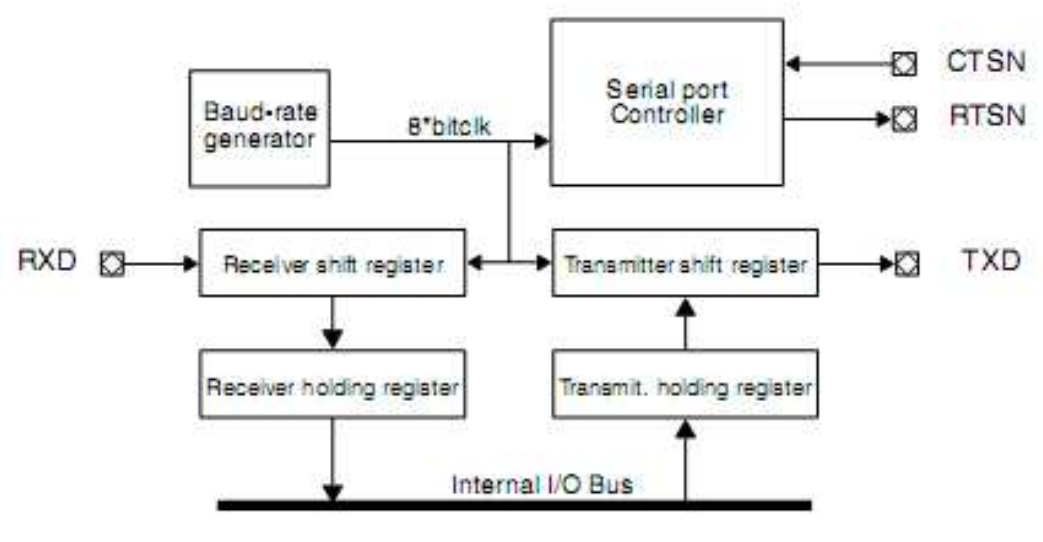

Figure 18 - UART block diagram

\subsubsection{Transmission}

The transmission is enabled through the TE bit in the UART control register. When ready to transmit, data is transferred from the transmitter holding register to the transmitter shift register and converted to a serial stream on the transmitter serial output pin (TXD). It automatically sends a start bit followed by eight data bits, an optional parity bit, and one stop bit (Figure 19). The least significant bit of the data is sent first.

\section{Data frame, no parity: $\quad \quad$\begin{tabular}{ll|l|l|l|l|l|l|l|l|} 
Start & $D 0$ & $D 1$ & $D 2$ & $D 3$ & $D 4$ & $D 5$ & $D 6$ & $D 7$ & Stop \\
\hline
\end{tabular}

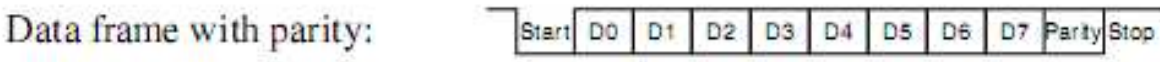

Figure 19 - UART data frames

Following the transmission of the stop bit, if a new character is not available in the transmitter holding register, the transmitter serial data output remains high and the transmitter shift register empty bit (TSRE) will be set in the UART control register. Transmission resumes and the TSRE is cleared when a new character is loaded in the transmitter holding register. If the transmitter is disabled, it will continue operating until the character currently being transmitted is completely sent out. The transmitter holding register cannot be loaded when the transmitter is disabled. If flow-control is enabled, the CTSN input must be low in order for the character to be transmitted. If it is deasserted in the middle of a transmission, the character in the shift register is transmitted and the transmitter serial output then remains inactive until CTSN is asserted again. 


\subsubsection{Reception}

The reception is enabled for data reception through the receiver enable (RE) bit in the UART control register. The receiver looks for a high to low transition of a start bit on the receiver serial data input pin. If a transition is detected, the state of the serial input is sampled a half bit clocks later. If the serial input is sampled high the start bit is invalid and the search for a valid start bit continues. If the serial input is still low, a valid start bit is assumed and the receiver continues to sample the serial input at one bit time intervals (at the theoretical centre of the bit) until the proper number of data bits and the parity bit have been assembled and one stop bit has been detected. The serial input is shifted through an 8bit shift register where all bits have to have the same value before the new value is taken into account.

During reception, the least significant bit is received first. The data is then transferred to the receiver holding register (RHR) and the data ready (DR) bit is set in the UART status register. The parity, framing and overrun error bits are set at the received byte boundary, at the same time as the receiver ready bit is set. If both receiver holding and shift registers contain an unread character when a new start bit is detected, then the character held in the receiver shift register will be lost and the overrun bit will be set in the UART status register. If flow-control is enabled, then the RTSN will be negated (high) when a valid start bit is detected and the receiver holding register contains an unread character. When the holding register is read, the RTSN will automatically be reasserted again.

\subsubsection{Loop back}

If the LB bit in the UART control register is set, the UART will be in loop back mode. In this mode, the transmitter output is internally connected to the receiver input and the RTSN is connected to the CTSN. It is then possible to perform loop back tests to verify operation of receiver, transmitter and associated software routines. In this mode, the outputs remain in the inactive state, in order to avoid sending out data.

\subsection{Functionalities verified}

Taking in account the brief specification of the UART module above, the following functionalities were selected to be check: data transfer (transmission/reception), flowcontrol and loop back. 
The test cases were written using the HAL generator functions, compiled to Leon2 processor architecture and performed their execution on the platform. For a consistent results analysis, the test cases were also re-written using the same programmer language (C) without the support of the HAL generator functions. So the results analysis was done comparing the effort to perform the functional verification. Both $\mathrm{C}$ codes can be seen in Appendix C. In sequence the results' analysis are shown.

\subsection{Results Analysis}

We start the results analysis discussing if the HAL generator is functional, that is, are its functions capable of accessing and manipulating the registers and its fields correctly? In second moment we discuss the actual benefits of the HAL generator in a $\mathrm{SoC}$ verification flow.

To check if the HAL generator is functional, the simulation results of the two set of test cases (provided by Gaisler and written using HAL functions) were compared and showed that the uarts' registers values appeared in line with the expected, therefore allowing us to conclude that the test case was successful. This first result guarantees that the functions generated by HAL are able to access and manipulate the registers values according to the system requirements. Hence we can affirm that the HAL generator is functional.

The HAL generator is functional; however its main contribution in the SoC functional verification flow is supporting the verification engineers to perform the verification spending less time than usual. With the support of the HAL functions in C coding test cases, the verification becomes easier than usual. For instance, in the uarts test cases examples, $133 \mathrm{C}$ code lines were needed to build the test cases without the aid of the HAL functions (Gaisler test cases). With the aid of the HAL functions, this number was reduced to $83 \mathrm{C}$ code lines. This gain is due to the fact that the addresses maps define file, macros and/or masks and/or structures needed to access the registers and their fields are implemented by the HAL. The complexity and effort of this work is overcome by the HAL functions.

Comparing the time spent to write the tests cases (write the $\mathrm{C}$ code and perform unit test), execute and conclude the functional verification we experienced a gain of $33 \%$ of time saved using the HAL functions. To perform the functional verification for those functionalities using the HAL generator were needed one man power during two days. Without the support of the HAL functions three days were needed. 
The HAL also guarantees a code right-by-construction as the masks values, used to manipulate the registers and fields, are taken from the IP-XACT description, calculated and applied to the functions. When the verification engineers calculate the masks values and write their own methods to access and manipulate the registers and fields by hand, they are susceptible to making mistakes during the code writing. Delays during the functional verification are caused due to mistakes in test cases coding.

Another important contribution around the use of HAL functions consists in gain of readability and meaningful test case codes. Once the HAL applies its functions' nomenclatures to the component, registers and field names, it becomes easier to understand the function of each code line. Figure 20 shows the function documentation automatic generated by the HAL generator. The value of the element description from the IP-XACT SoC description is automatically captured and used in the function documentation. In the nomenclature of the function is possible to identify the function behavior, the IP manipulated, the register and its field accessed.

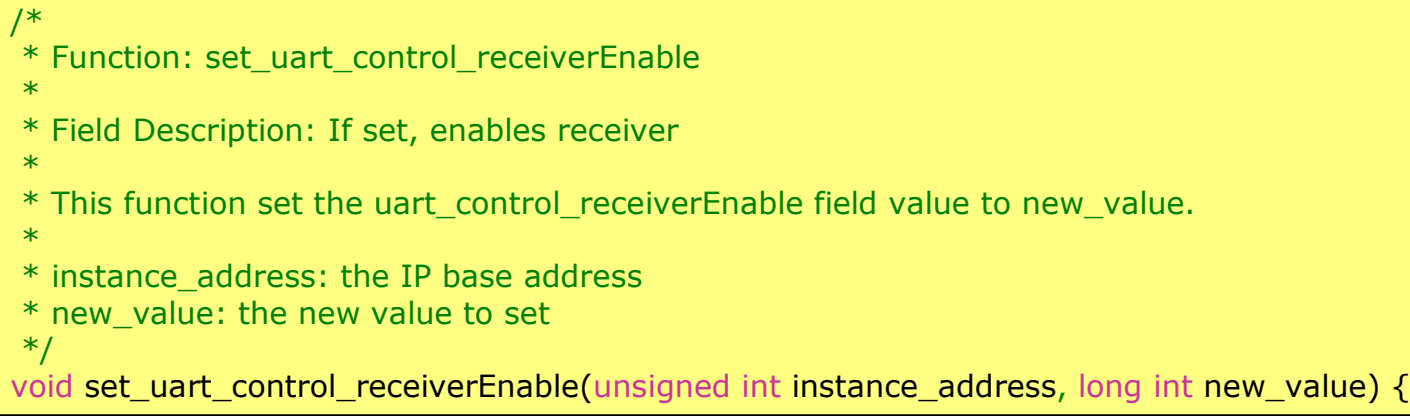

Figure 20 - Functions documentation and nomenclature

Figure 21 shows two small pieces of the $C$ test cases codes written. Both perform the same action, enabling the uarts (uart_1 and uart_2) to transmit and receive data, and checking if the transmission hold field is empty. The code written using HAL functions is shown on the left-hand side. As we can see the code written using the HAL functions is more understandable than the other one. The HAL functions also allow an abstraction of pointers manipulation, once each function is called through a simple local method with primitive type arguments. The pointer manipulation is in charge of the HAL functions. 


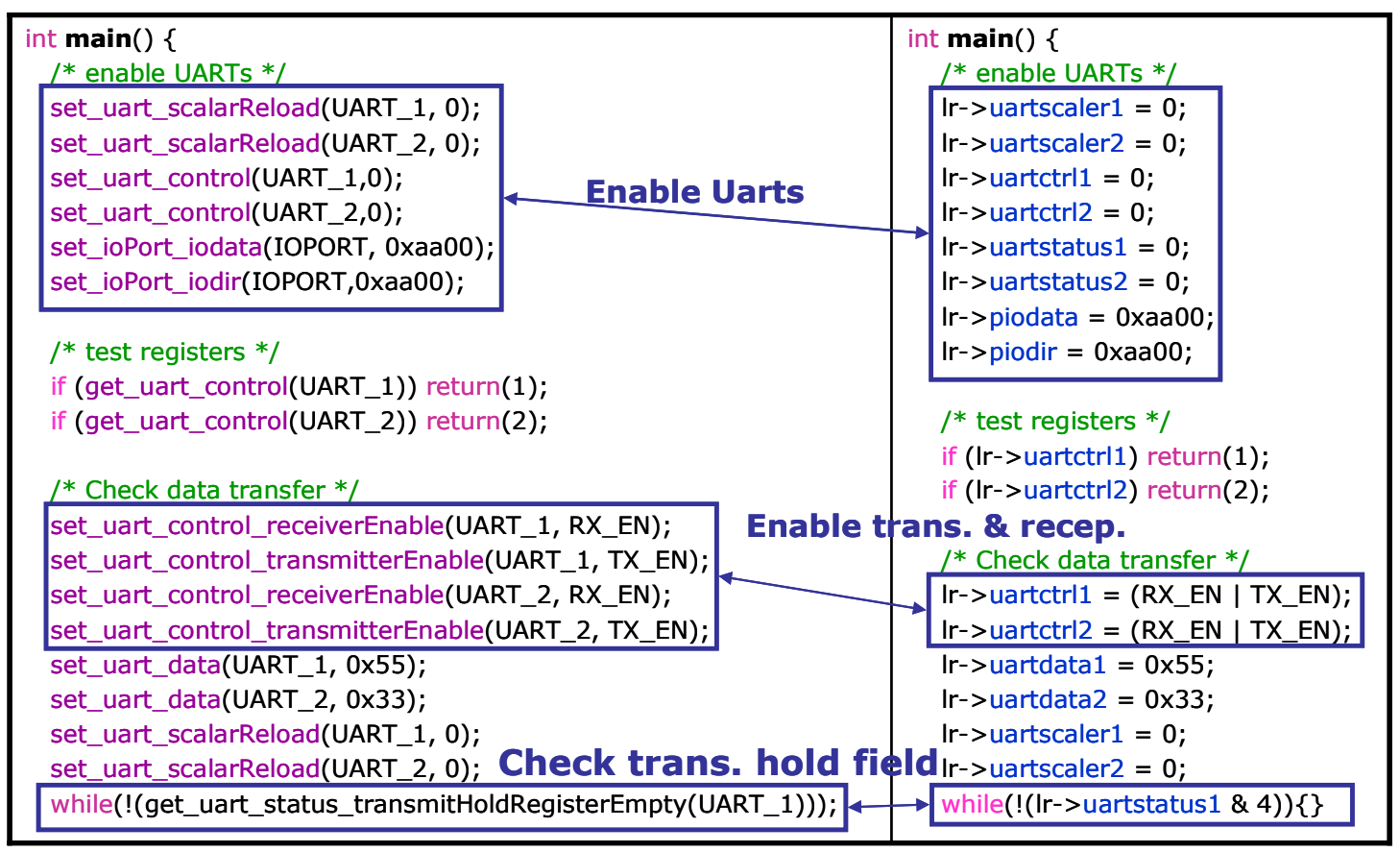

Figure 21 - Test case codes analysis

Test case reuse is also empowered by HAL generator. For each SoC, the HAL generator captures the IPs base addresses and creates their registers and field access functions. For instance, suppose a test case already written for an IP found in a SoC architecture; if this IP is used in another SoC context, the same test case can be easily reused just by keeping the IP instances nomenclature in the IP-XACT description.

Finally, the structured and well documented code generated by HAL when integrated to Eclipse tool, offers a powerful development environment once Eclipse's features like word completion, content assist, parameter hints, among others, provide a highly productive coding activity.

\subsection{Final Considerations}

This Section presented the use of the HAL generator tool in a SoC functional verification.

This case study was fundamental to evaluate the HAL generator inside the SAVE methodology. The tool was well integrated with the Magillem Design Environment and capable to manipulate the SoC IP-XACT description.

The initial idea was use the SoC platform example released in IP-XACT 1.4 version. However the platform doesn't work as defined in its manual. The platform is based on Leon2 processor and according its user manual two configurations could be used. The first one uses a bus functional model of the Leon2 processor that doesn't enables the execution 
of binary codes. The second configuration uses a real Leon2 processor which should be capable to execute binary codes compiled to Leon2 target. Once the intent of the case study was running $\mathrm{C}$ binary codes the second configuration was chosen. During the work we noticed that the results don't appears in line with expected so we decided debug the platform. After two weeks and few contacts with the IP-XACT maintainers we identified that the configuration using the real Leon2 processor doesn't work properly. To overcome this problem we decide to use the platform delivered by Gaisler Research Group that is also based on Leon2. The platform was ported to Magillem Design Environment and used in alternative instead the example provided by IP-XACT.

Solved the problem with the platform example the functional verification could be performed. The results analysis showed that the use of the HAL functions saved $33 \%$ of time comparing with the flow without the support of the tool. The test cases written with the aid of the HAL functions are more readable. The HAL functions documentation automatically generated by the tool also offers meaningful codes. Another benefit brought by the HAL functions consists of the reuse of $\mathrm{C}$ test cases. The HAL generator can be used in any SoC context, when an IP appears in various SoC the same test case can be used just keeping the instance name of such IP unchanged.

In next Section the finals conclusions of this work are presented, as well the future works. 


\section{Conclusions}

This work investigated and proposed a tool to automate the Hardware Abstract Layer generation in a SoC functional verification. The tool proposed is part of the Spirit Assisted Verification Environment (SAVE). SAVE defines a set of tools to support the functional verification of System-on-Chip. These tools are used to handles the tasks involved on SoC functional verification in an automatic manner.

\subsection{Main Contributions}

As presented in the Results Analysis Section, the proposed tool allows 33\% of reduction in total time of the functional verification. From the results comparison between the test case coding task with and without HAL functions, the quality of the code and the performance reached, we conclude that the HAL generator improves this task in the following aspects:

- Fast and easier way to perform the functional verification: the use of the HAL functions reduced the total time of the functional verification in $33 \%$. The HAL functions are well documented and structured becoming easier and intuitive coding the $\mathrm{C}$ test cases;

- Functions right-by-construction (bugs free): once the HAL API implementation is done by the tool we have codes right-by-construction reducing the possibility of the engineer make mistakes coding it by hand;

- More meaningful codes and consequent maintainability gain: the HAL functions are well documented. All fields and registers description are imported from the IP-XACT description giving useful information to the engineer when coding the test cases;

- Test case reuse: once the HAL generator can be used in any SoC context the reuse of test cases is empowered. When the same IP is used in two different SoC to re-use its test cases the unique constraint is keep the instance name unchanged in the IP-XACT description;

- Highly productive coding environment when integrated with Eclipse IDE: integrated with Eclipse IDE the HAL functions documentation is easily visualized and the $\mathrm{C}$ coding is supported by the IDE features like word completion, content assist, parameter hints, among others. 
The aspects listed above show that the HAL generator offers a set of significant gains in $\mathrm{SoC}$ functional verification.

\subsection{Difficulties Met}

The difficulties met throughout this work were managed considering the best way to overcome them. Difficulties regarding tool limitations, like Magillem DE, were set up as HAL constraints and should be completely solved with new Magillem versions. Regarding the main HAL generator constraints, we can list:

- bankedBank elements are not supported because Magillem does not manage this kind of element. This implies that addressBlock elements contained in bankedBank elements are not accessible by HAL functions.

- Remap State is not taken into account because Magillem does not support IPXACT attributes types. Once the remapAddress element in Mirrored Slave Interface is configurable by attributes, it is not possible to manage the bus interface state without accessing the target attributes values. For all remapAddress elements found in Mirrored Slave Interface, just the default state is considered.

- Configurable Element is not supported. The "bind" between configurable elements and their elements targets (bus interface, memory, register, field, etc) is given by attributes values. As Magillem does not support it, this functionality could not be implemented.

The platform model used to test the HAL generator also presented unexpected problems. The SPIRIT Consortium provides a platform example ready to be used. However this platform does not offer the minimal features to test and validate the HAL generator. Although this platform is based on the well known Leon2 processor, it does not use the core processor. Instead of a Leon 2 core processor they put in place a bus functional model that does not work as a real processor model. As a consequence, another platform needed to be used to test and validate the HAL generator. In that case, to overcome this problem the Leon2 platform provided by Gaisler Research was taken and a new IP-XACT design configuration was created for it. This change allowed the HAL generator to be tested and validated. 


\section{3. $\quad$ Future Works}

The HAL generator is a powerful tool but two weaknesses should be considered in future work.

Even that the tool allows a time reduction of $33 \%$ in the functional verification, the time consumption in the test cases execution using the HAL functions increase. As each register or field access consists in functions calls, the tests cases execution time is longer than the test cases written without the aid of the HAL functions. In the uarts tests cases example, execution took about three times longer. This issue was expected since the beginning of the work due to the many bus activity performed by the HAL functions. In a first moment the aiming was investigated if the HAL functions could be generated in an automatic manner using the IP-XACT standard and could reduce the total time of the functional verification. Considering that the simulation time is insignificantly compare with the all time needed to perform the functional verification we can affirm that this issue doesn't commits the results of the tool.

A strategy to overcome the test cases' execution time problem is known and will be implemented in newer HAL generator versions. In the new versions the calls should contain some sort of caching mechanism that will remember the value read from or written to the given register. With this functionality the time of execution will be shorter.

Due to the problem with the initial platform example adopted the project delayed in one month committing the case study. Another future work consists in applying the tool in others SoC platform to validate the tool in a more wide context and also to identify points to improvement. 


\section{References}

[1] Advanced High-performance Bus - AMBA. Available in: $<$ http://www.arm.com/products/solutions/AMBAHomePage.html > Accessed in: $18^{\text {th }}$ November 2008.

[2] Cadence Functional Verification Kit for ARM processor. Available in: $<$ http://w2.cadence.com/datasheets/arm_func_ver_kit.pdf $>$ Accessed in: 19 ${ }^{\text {th }}$ March 2009 .

[3] Dahan, A.; Geist, D.; Gluhovsky, L.; Pidan, D.; Shapir, G.; Wolfsthal, Y.; Benalycherif, L.; Kamidem, R.; Lahbib, Y. "Combining system level modeling with assertion based verification". Quality of Electronic Design, 2005. ISQED 2005. Sixth International Symposium on. 2005.

[4] Deaton, Craig; Littlefield, Jay. "Easy Structured Verification Planning Unsing GamePlan Verification Planner". Jasper Design Automation. August 2006. Available in: <www.jasper-da.com> Accessed in: $18^{\text {th }}$ November 2008.

[5] Eclipce.org. Available in: <www.eclipse.org> Accessed in: $18^{\text {th }}$ November 2008.

[6] Extensible Markup Language - XML. Available in: 〈www.w3.org/XML> Accessed in: $19^{\text {th }}$ November 2008.

[7] Hunsinger, F.; Francois, S.; Jerraya, A.A. "Definition of a systematic method for the generation of software test programs allowing the functional verification of System On Chip (SoC)". In Proceedings of the 4th International Workshop on Microprocessor Test and Verification: Common Challenges and Solutions. 2003.

[8] Gaisler Research. Available in: <www.gaisler.com> Accessed in: $23^{\text {rd }}$ September 2008.

[9] Janick Bergeron. Writing Testbenches - Functional verification of HDL models, Springer Science, 2003.

[10] Janick Bergeron. Writing Testbenches Using System Verilog. Massachusetts: Springer, 2006.

[11] Karina Silva, Elmar Melcher, et al. "An automatic testbench generation tool for a systemc functional verification methodology". In Symposium on Integrated Circuits and Systems Design, 2004.

[12] Kenney, Jim. "Using a processor-driven testbench for functional verification of embedded SoCs". Embedded.com. $4^{\text {th }}$ October 2006. Available in: <http://www.embedded.com/columns/showArticle.jhtml?articleID=193104351>. Accessed in: $26^{\text {th }}$ January 2009.

[13] Klein, R.; Piekarz, T., "Accelerating functional simulation for processor based designs". System-on-Chip for Real-Time Applications, 2005. Proceedings. Fifth International Workshop on. 2005. 
[14] Komarnitzky, Arie; Ben-Ezer, Nadav; Lyubinsky, Eugene. "Unique Approach to Verification of Complex SoC Designs". Design \& Reuse Industry Articles. 2006. Available in: <http://www.design-reuse.com/articles/12496/unique-approach-toverification-of-complex-soc-designs.html>. Accessed in: $26^{\text {th }}$ January 2009.

[15] Kruijtzer, W., van der Wolf, P., de Kock, E., Stuyt, J., Ecker, W., Mayer, A., Hustin, S., Amerijckx, C., de Paoli, S., and Vaumorin, E. "Industrial IP integration flows based on IP-XACT ${ }^{\mathrm{TM}}$ standards". In Proceedings of the Conference on Design, Automation and Test in Europe. 2008.

[16] Kwanghyun Cho; Jaebeom Kim; Euibong Jung; Sik Kim; Zhenmin Li; Young-Rae Cho; Byeong Min; Kyu-Myung Choi, "Reusable platform design methodology for SoC integration and verification". SoC Design Conference, 2008. ISOCC '08 International, 2008.

[17] Magillem Design Services. Magillem Design Environment. Available in: <http://www.magillem.com> Accessed in: $18^{\text {th }}$ November 2008.

[18] Mishra, P.; Mingsong Chen, "Efficient Techniques for Directed Test Generation Using Incremental Satisfiability". VLSI Design, 2009 22nd International Conference on. 2009.

[19] Roy, S. K., Ramesh, S., Chakraborty, S., Nakata, T., and Rajan, S. P. "Functional Verification of System on Chips-Practices, Issues and Challenges". In Proceedings of the 2002 Conference on Asia South Pacific Design automation/VLSI Design. 2002.

[20] STMicroelectronics. Available in: 〈www.st.com>. Accessed in: $19^{\text {th }}$ November 2008.

[21] The Spirit Consortium. Available in: 〈www.spiritconsortium.org > Accessed in: $18^{\text {th }}$ November 2008.

[22] The Spirit Consortium. IP-XACT 1.4 specification. $12^{\text {th }}$ March 2008. Available in: 〈www.spiritconsortium.org/releases/1.4>. Accessed in: $19^{\text {th }}$ November 2008.

[23] The Spirit Consortium General Meeting at DAC 2008. $\mathbf{4 5}^{\text {th }}$ Design Automation Conference. $\quad 9^{\text {th }} \quad$ June $2008 . \quad$ Available in: $<$ http://www.spiritconsortium.org/press/presentations/20080609_SPIRIT_DAC_08_fin al.pdf $>$. Accessed in: $18^{\text {th }}$ November 2008.

[24] Yoo, S. and Jerraya, A. A. "Introduction to Hardware Abstraction Layers for SoC". In Proceedings of the Conference on Design, Automation and Test in Europe. 2003.

[25] Ziv, Avi. "Functional Verification and the SoC Challenge". IBM Research Lab. 2003. Available in: <www.mpsoc-forum.org/2003/slides/soc_verification.pdf>. Accessed in: $18^{\text {th }}$ November 2008. 


\section{Appendix A - IP-XACT Standard}

The IP-XACT Standard [22] is the official name for the meta-data and API standards delivered by the SPIRIT Consortium. The standard describes an eXtensible Markup Language (XML) [6] data format and structure, documented with a schema for capturing the meta-datawhich documents the design of the intellectual property used in the development, implementation, and verification of electronic systems.

The standard also includes a Tight Generator Interface (TGI) to provide consistent, tool-independent access to the meta-data. The XML documents described and validated by the schema comprise a standard method to document IP that is compatible with automated integration techniques. The TGI provides a standard method for linking generation tools into a system development framework, enabling a flexible Design Environment (DE). The design environment enables the designer to work with IP-XACT design IP through a coordinated front-end and IP design database. Figure 22 shows an IP-XACT design environment overview. The top-level schemas showed in Figure 22 are described in sequence.

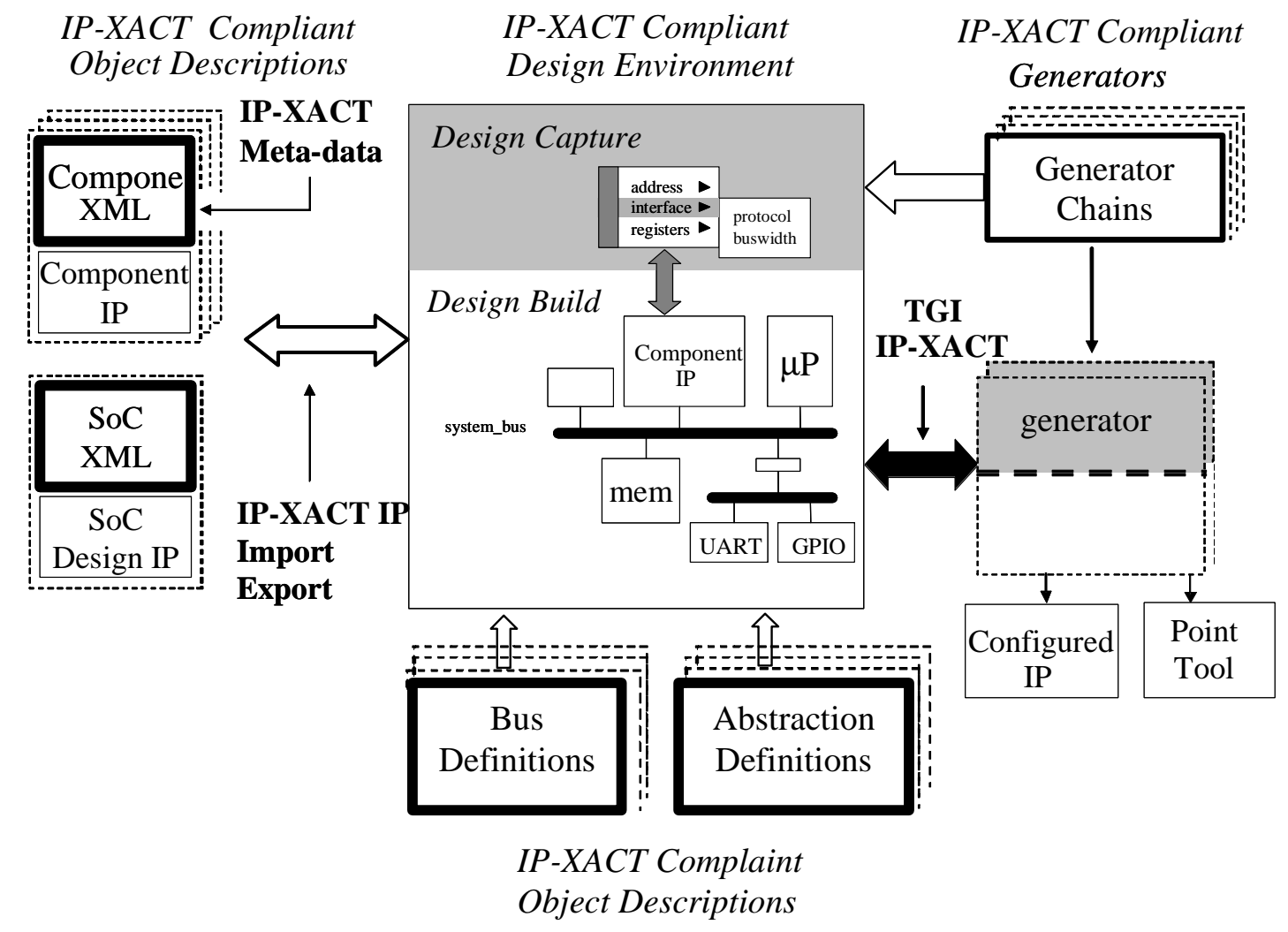

Figure 22 - IP-XACT design environment [22] 
The IP-XACT defines seven top-level schemas. Each schema definition can be used to create object descriptions of the corresponding type:

- A bus definitions description defines the type attributes of a bus. It describes the high-level aspects of a bus or interconnections. It contains the following elements:

a) directConnection (mandatory) specifies what connections are allowed. The directConnection element is of type Boolean. A value of true specifies these interfaces may be connected in a direct master to slave fashion. A value of false indicates only non-mirror to mirror type connections are allowed (i.e., master-mirroredMaster, slavemirroredSlave, or system-mirroredSystem);

b) isAddressable (mandatory) specifies the bus has addressing information. The isAddressable element is of type Boolean. A value of true specifies these interfaces contain addressing information and a memory map can be traced through this interface. A value of false indicates these interfaces do not contain any traceable addressing information;

c) extends (optional) specifies if this definition is an extension from another bus definition;

d) maxMasters specifies the maximum number of masters that are allowed on the bus. If the maxMasters element is not present, the numbers of masters is unbounded;

e) maxSlaves specifies the maximum number of slaves that are allowed to appear on the bus. If the maxSlaves element is not present, the numbers of slaves is unbounded;

f) description (optional) allows a textual description of the interface;

g) vendorExtensions (optional) contains any extra vendor-specific data related to the interface.

- An abstraction definition description defines the representation attributes of a bus. It describes the low-level aspects of a bus or interconnection. It contains the following elements: 
a) busType (mandatory) specifies the bus definition that this abstraction definition;

b) extends (optional) specifies if this definition is an extension from another abstraction definition;

c) ports (mandatory) is a list of logical ports;

d) description (optional) allows a textual description of the interface;

e) vendorExtensions (optional) contains any extra vendor-specific data related to the interface;

- A component description defines an IP or interconnect structure. An IP-XACT component is the central placeholder for the objects meta-data. Components are used to describe cores (processors, co-processors, DSPs, etc.), peripherals (memories, DMA controllers, timers,UART, etc.), and buses (simple buses, multi-layer buses, cross bars, network on chip, etc.). An IP-XACT component can be of two kinds: static or configurable. A DE cannot change a static component. A configurable (or parameterized) component has configurable elements (such as parameters) that can be configured by the DE and these elements may also configure the RTL or TLM model. An IP-XACT component can be a hierarchical object or a leaf object. Leaf components do not contain other IP-XACT components, while hierarchical components contain other IPXACT sub-components. This can be recursive by having hierarchical components that contain hierarchical components, etc.-leading to the concept of hierarchy depth. The IP being described may have a completely different hierarchical arrangement in terms of its implementation in RTL or TLM to that of its IP-XACT description. So, a description of a large IP component may be made up of many levels of hierarchy, but its IP-XACT description need only be a leaf object as that completely describes the IP. On the other hand, some IP can only be described in terms of a hierarchical IP-XACT description, no matter what the arrangement of the implementation hierarchy. An IP-XACT component may contain a channel or a bridge. A channel is a special IP-XACT object that can be used to describe multi-point connections between regular components that may require some interface adaptation. A bridge is a point-topoint reference of slave to master interfaces. Both of these concepts are used to 
describe the interconnect between components. Among others it contains the following main elements:

a) busInterfaces (optional) specifies all the interfaces for this component. A busInterface is a grouping of ports related to a function, typically a bus, defined by a bus definition and abstraction definition;

b) channels (optional) specifies the interconnection between interfaces inside of the component;

c) remapStates (optional) specifies the combination of logic states on the component ports and translates them into a logical name for use by logic that controls the defined address map;

d) addressSpaces (optional) specifies the addressable area as seen from busInterfaces with an interface mode of master or from cpus;

e) memoryMaps (optional) specifies the addressable area as seen from busInterfaces with an interface mode of slave;

f) model (optional) specifies all the different views, ports, and model configuration parameters of the component;

g) componentGenerators (optional) specifies a list of generator programs attached to this component;

h) cpus (optional) indicates this component contains programmable processors;

i) description (optional) allows a textual description of the component;

j) vendorExtensions (optional) contains any extra vendor-specific data related to the interface.

- A design description defines the configuration of and interconnection between components. A design describes a list of components referenced by this description, their configuration, and their interconnections to each other. The interconnections may be between interfaces or between ports on a component. A design description is analogous to a schematic of components. While a design description, with referenced components and interconnections, describes most of the information for a design, some information is missing, such as the exact port names used by a bus interface. To resolve thisa component 
description (referred to as a hierarchical component) is used. This component description contains a view with a reference to the design description. Together, the component and referenced design description form a complete single-level hierarchical description. From this point, it is simple to create additional hierarchical descriptions by including hierarchical component description in design descriptions. It contains the following elements:

a) componentInstances (optional) contains the list of components that are instantiated (referenced) inside the design;

b) interconnections (optional) contains the list of connections between bus interfaces of components listed inside the design;

c) adHocConnections (optional) contains a list of connections between component ports listed inside this design;

d) hierConnections (optional) contains a list of connections between a component instance's active bus interface and a bus interface inside the encompassing component. This element only allows making hierarchical reference between bus interfaces;

e) description (optional) allows a textual description of the component;

f) vendorExtensions (optional) contains any extra vendor-specific data related to the interface.

- An abstractor description defines an adaptor between interfaces of two different abstractions. Designs that incorporate IP models using different interface modeling styles (e.g., TLM and RTL modeling styles) may contain interconnections between such component interfaces using different abstractions of the same bus type. An IP-XACT description may describe how such interconnections are to be made using a special-purpose object called an abstractor. An abstractor is used to connect between two different abstractions of the same bus type (e.g., an APB_RTL and an APB_TLM). An abstractor shall only contain two interfaces, which shall be of the same bus definition and different abstraction definitions. Among others it contains the following main elements:

a) busType (mandatory) specifies the bus definition this bus interface references; 
b) abstractorMode (mandatory) determines the mode of the two interfaces contained. It can be master, slave, direct or system;

c) abstractorInterfaces (mandatory) are interfaces having the same bus type, but differing abstraction types;

d) model (optional) specifies all the different views, ports, and model configuration parameters of the abstractor;

e) abstractorGenerators (optional) specifies a list of generator programs attached to this abstractor;

f) description (optional) allows a textual description of the component;

g) vendorExtensions (optional) contains any extra vendor-specific data related to the interface.

- A generator chain description defines the grouping and ordering of generators. In IP-XACT, a design flow can be represented as a generator chain. A generator chain is an ordered sequence of named tasks. Each named task can be represented as a single generator or as another generator chain. This way, design flow hierarchies can be constructed and executed from within a given DE. The DE itself is responsible for understanding the semantics of the specified chain described in the generator chain description. It contains the following elements:

a) generatorChainSelector (optional) is a selection criteria for selecting one or more generatorChains;

b) componentGeneratorSelector (optional) is a selection criteria for selecting one or more component generators;

c) generator (optional) defines the generator;

d) chainGroup (optional) is an unbounded list of names to which this chain belongs. The group names are referenced in the generatorChainSelector element and can be used to organize the inclusion of generators;

e) description (optional) allows a textual description of the component;

f) vendorExtensions (optional) contains any extra vendor-specific data related to the interface; 
- A design configuration description defines additional configuration information for a generator chain or design description. Design configuration information is useful when transporting designs between design environments and automating generator chain execution for a design, by storing information that would otherwise have to be re-entered by the designer. Among others it contains the following mandatory and options elements:

a) designRef (mandatory) specifies the design description for this design configuration;

b) generatorChainConfiguration (optional) is an unbounded list of configuration information associated with a generatorChain or a generator defined within a generatorChain;

c) interconnectionConfiguration (optional) is an unbounded list of information associated with interface interconnections;

d) description (optional) allows a textual description of the component;

e) vendorExtensions (optional) contains any extra vendor-specific data related to the interface.

The interactions between the objects detailed above are illustrated in Figure 23. The arrows $(\mathrm{A} \rightarrow \mathrm{B})$ illustrate a reference from one object to another (e.g., reference from object A to object B). 


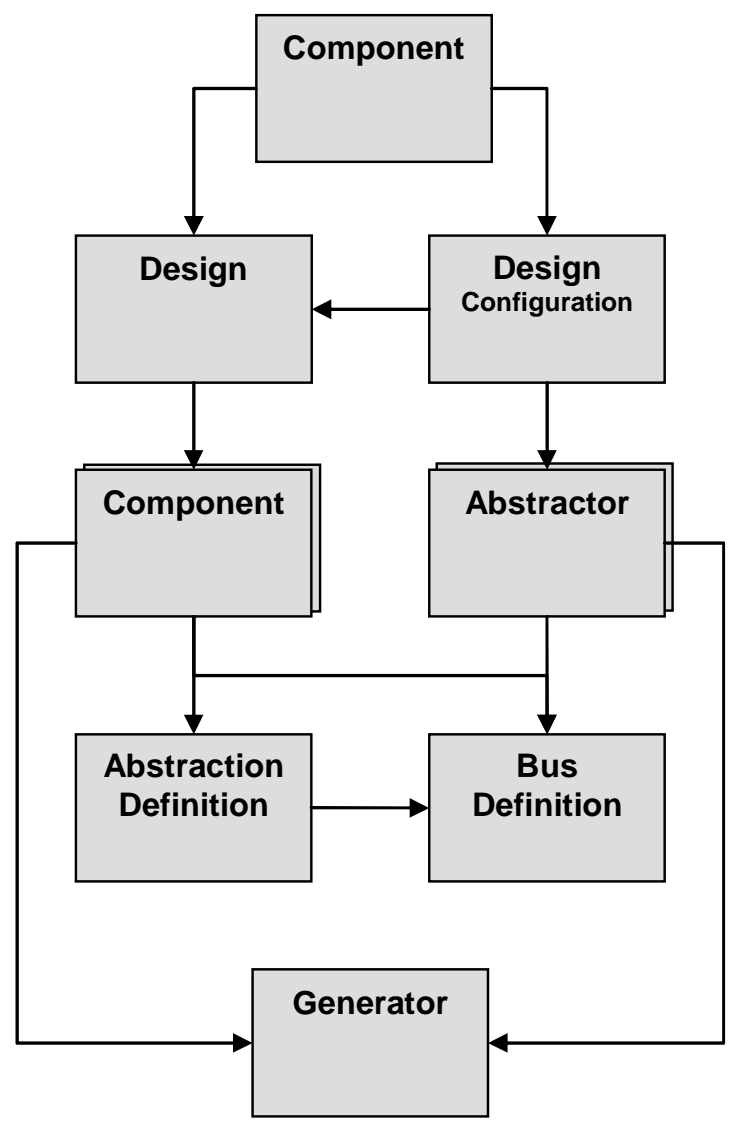

Figure 23 - IP-XACT objects interactions

\section{The IP-XACT generators}

The generators are executable objects (e.g., scripts or binary programs), which may be integrated within a design environment (referred to as internal) or provided separately as executable files (referred to as external). When executed, the generators perform queries or configure design descriptions and their related component and abstractor descriptions.

Internal generators use methods implemented by the design environment to access a design or component description and these methods are not defined by IP-XACT. External generators use the Tight Generator Interface (TGI) method to access a design or component description. The TGI describes an Application Program Interface (API), which provides the means of getting and setting values within the IP-XACT design currently represented in the design environment. In the Hardware Abstract Layer Generator section a detailed example of how TGI can be used is shown.

This document does not intend to address IP-XACT meta-data description in detail or to give a wide description of its objects once the standard document consists over three 
hundreds pages. The idea is to provide enough information for the understanding of the IP$\mathrm{XACT}$ role in this work.

In following an IP-XACT component description example for a Universal Asynchronous Receiver/Transmitter (UART) serial interface IP will be presented. The example is divided in four code regions.

The Figure 24 defines the IP-XACT version used in that description, the Vendor, Library, Name and Version (VLNV). Each IP has a unique VLNV this information is an ID for the IP.

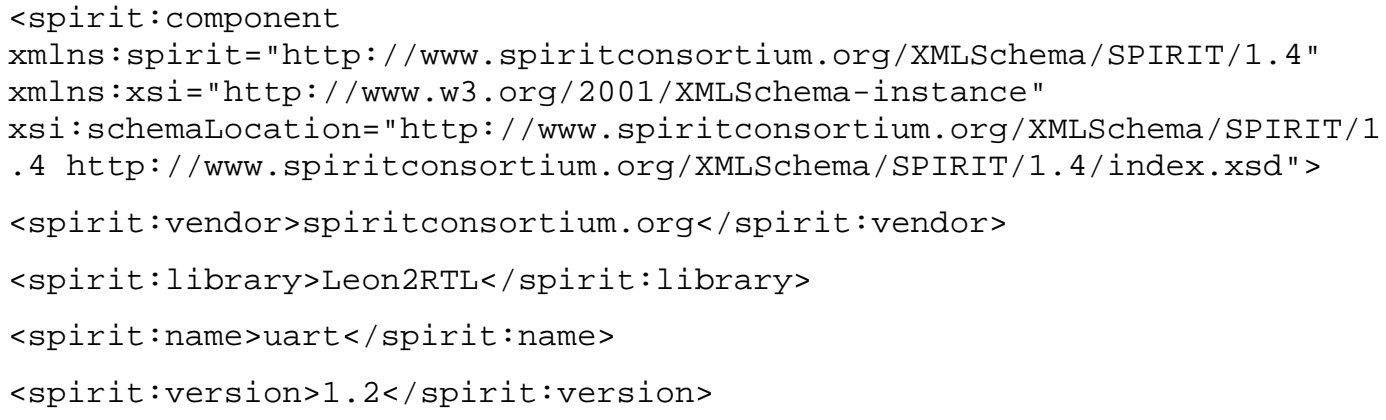

Figure 24 - Component definition

In the Figure 25 the busses interfaces are defined, these are used by the IP to communicate with the others modules. In that example we can see just one interface declared, the others interfaces were removed. In the example we can see the bus name, type and abstraction defined for interface. We can have different type of busses and each type can have one or more abstraction definition. The tag slave defines that the interface acts on the system as a slave interface. The tag portMap define' the connections between the ports defined in the abstraction and the IP physical ports.

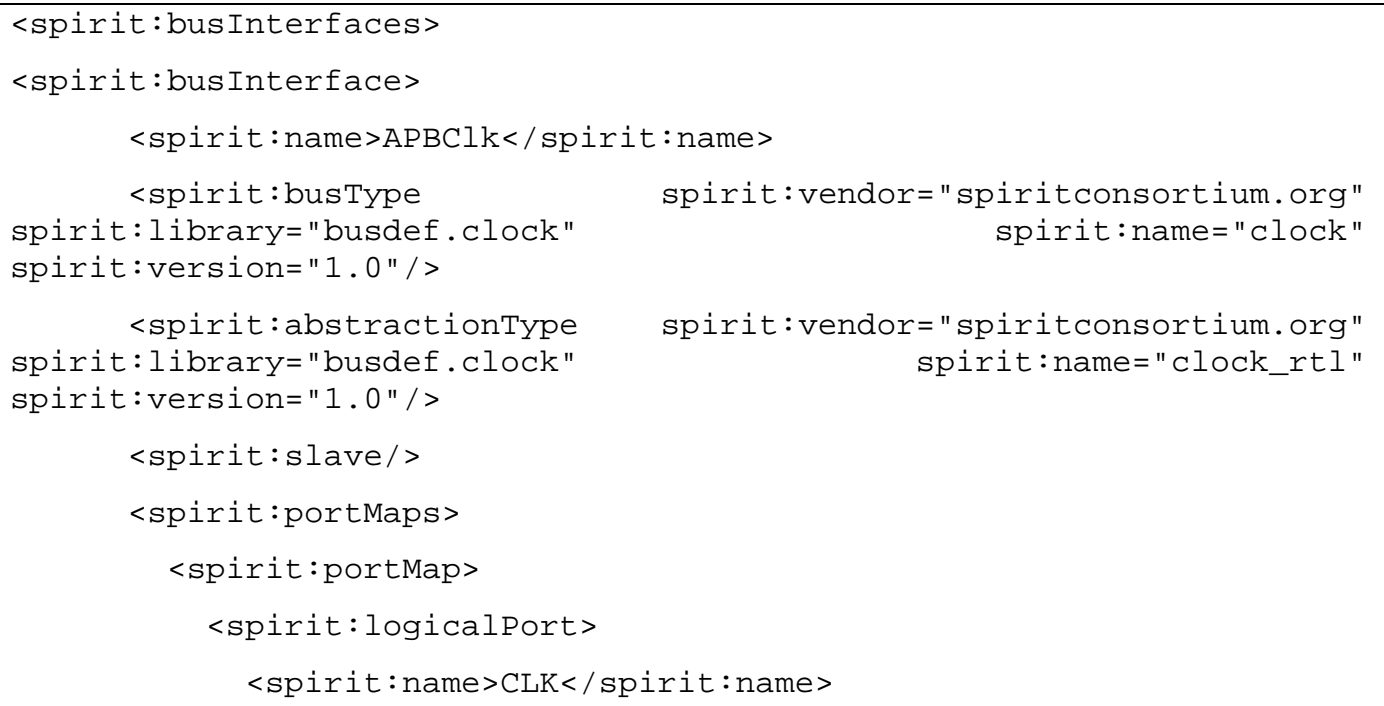




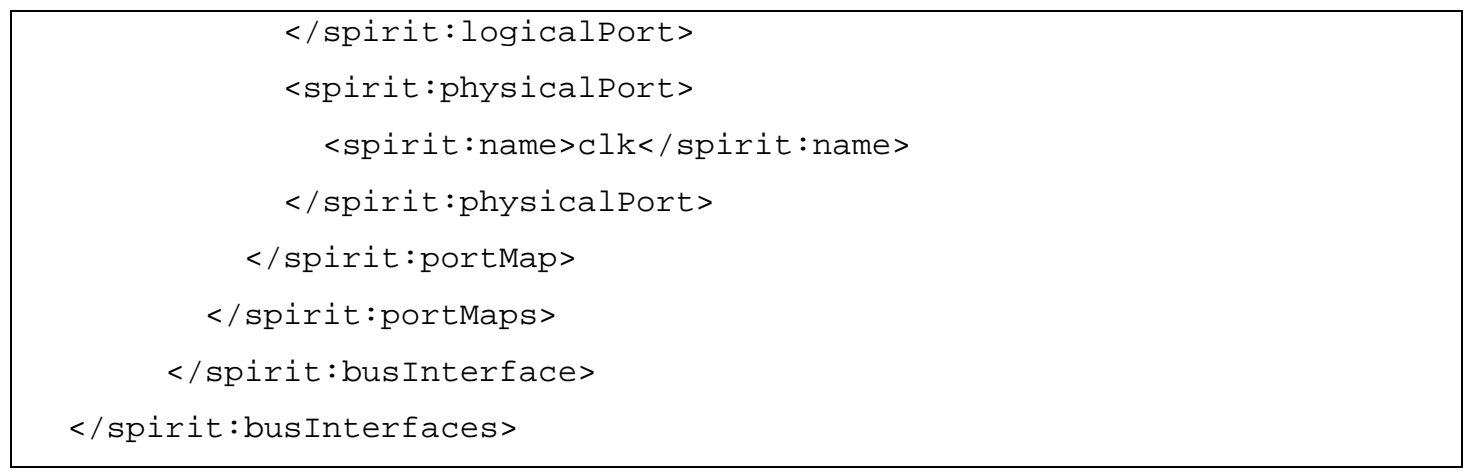

Figure 25 - Busses definition

In the Figure 26 we have the IP memory blocks representation. In the IP-XACT standard every register is part of a memoryMap, which can have one or more address blocks. Each AddressBlock can have one or more registers. The registers description tags are more detailed in Section 4.2.

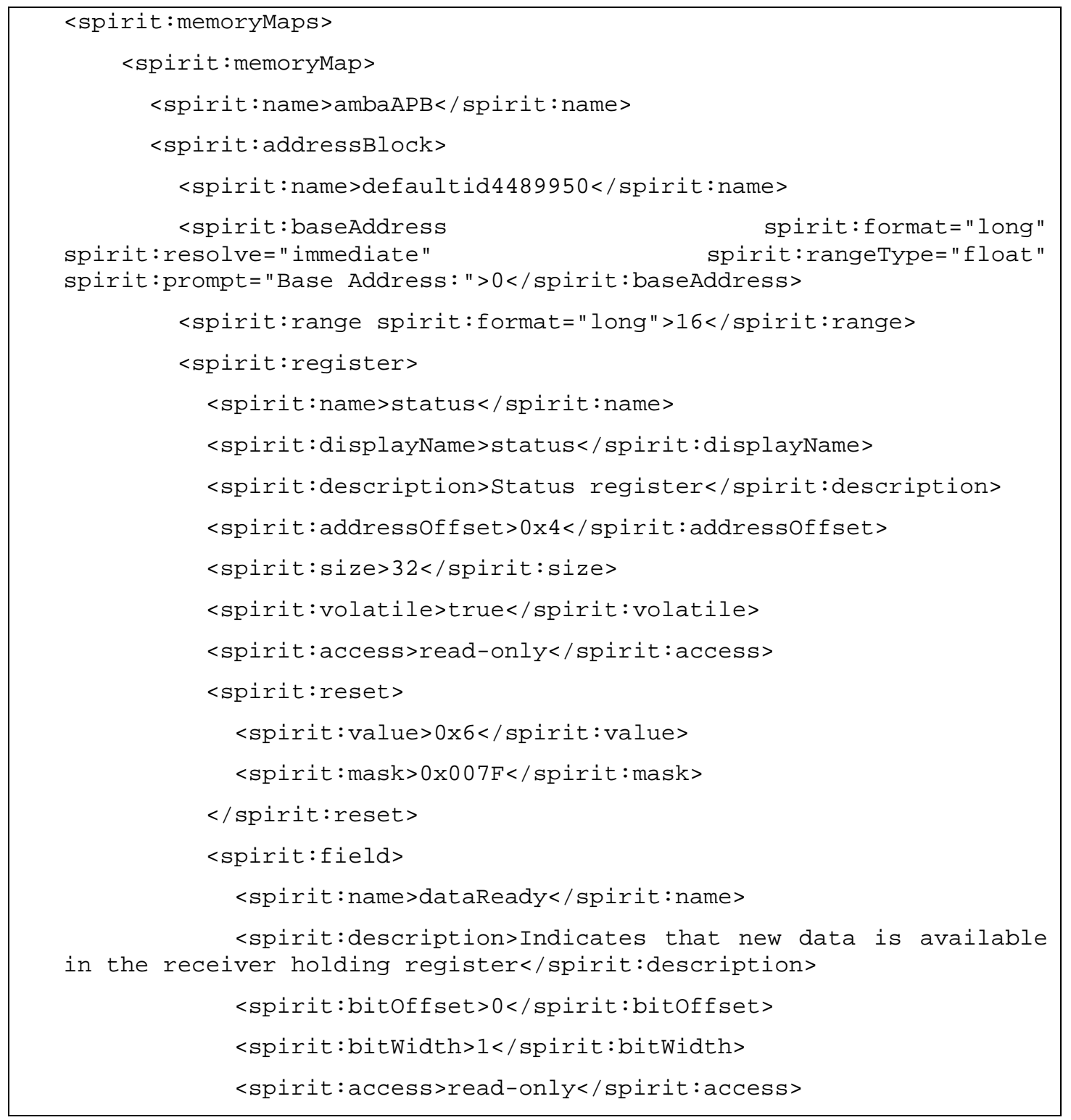




</spirit:field>
</spirit:register $>$
</spirit:addressBlock>
</spirit:memoryMap>
</spirit:memoryMaps>

Figure 26 - Memory maps, registers and fields definition

The Figure 27 defines the physical ports. The physical ports can be wire or transactional types. The wire represents ports in an RTL model. The transaction ports are used to represent complex structures used in high-level description like a transaction level model. The ports direction can be in, out or both.

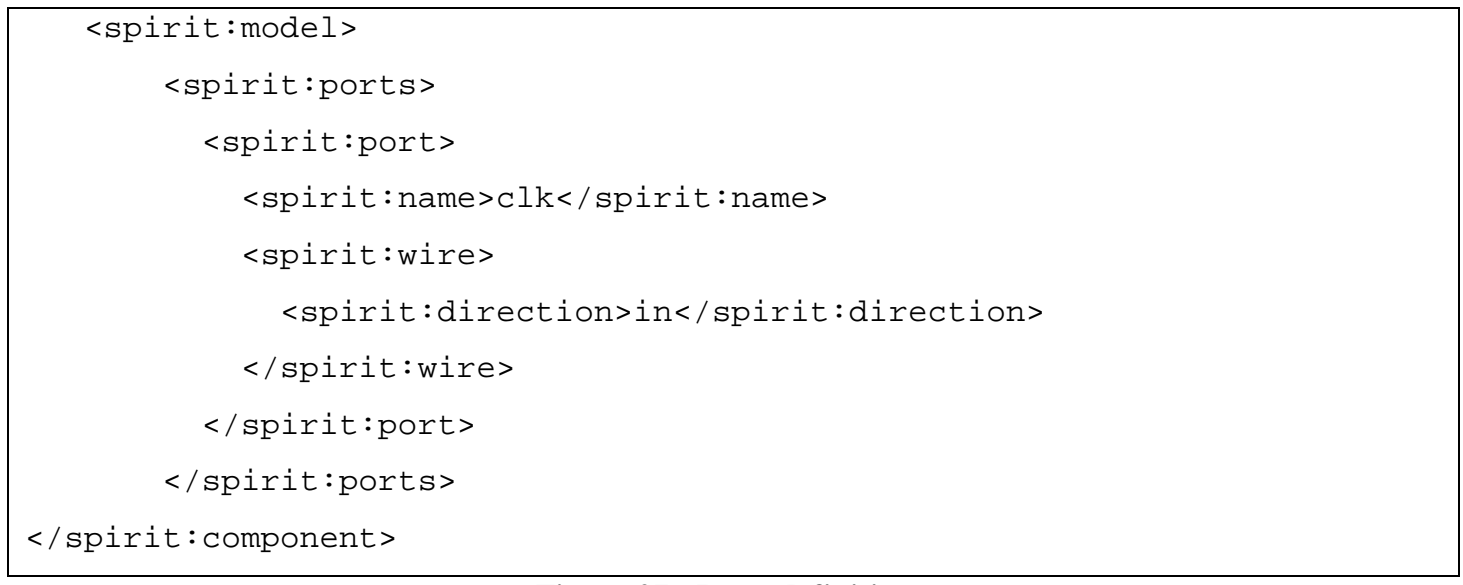

Figure 27 - Ports definition 


\section{Appendix B - Slave interfaces base address definition algorithm engine}

The Figure 28 presents a flow chart representing the algorithm to define the base address for each slave interface. The algorithm presupposes that the CPU master interface is known. The flow chart is presented using the IP-XACT nomenclature for busses interfaces. Just the macro activities are pointed. This algorithm is performed over the IPXACT SAVE representation of the DUV built during the process to create the HAL functions (Section 4.1). 


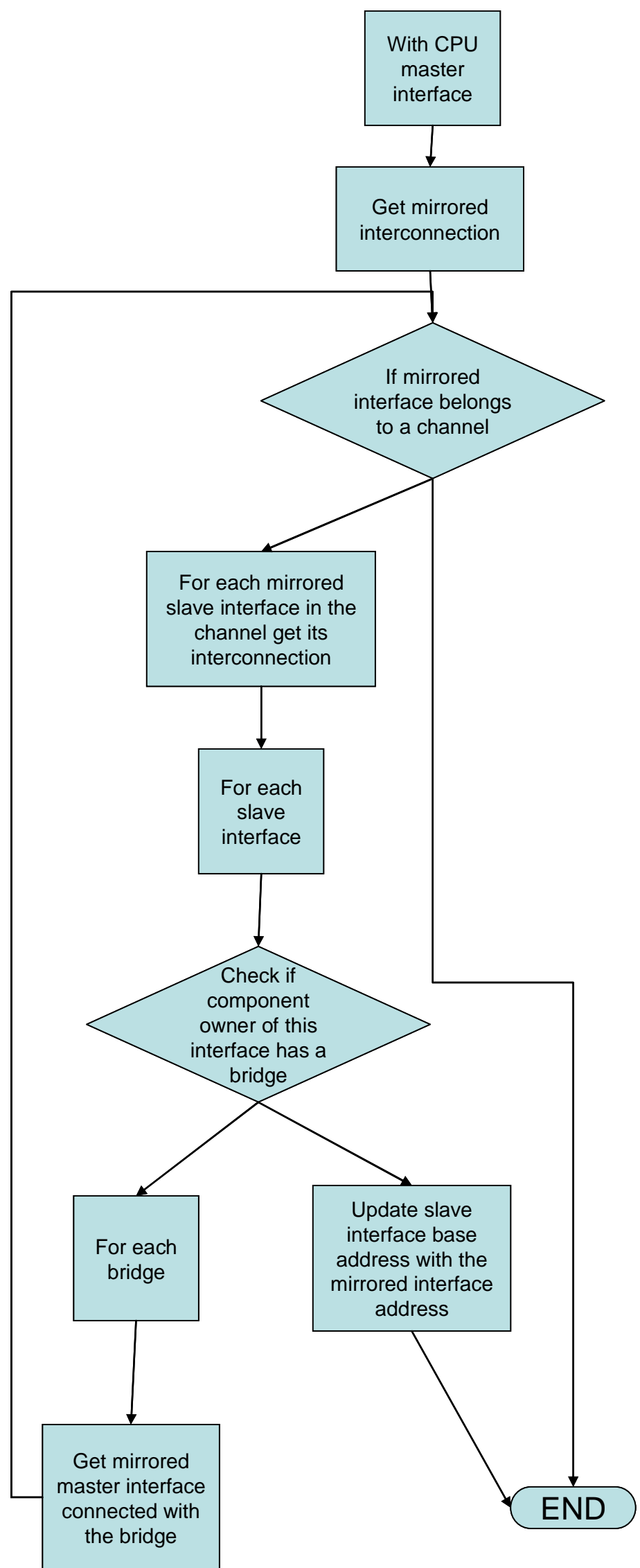

Figure 28 - Algorithm flow-chart 


\section{Appendix C - Uarts test cases codes}

Test cases written using HAL functions calls.

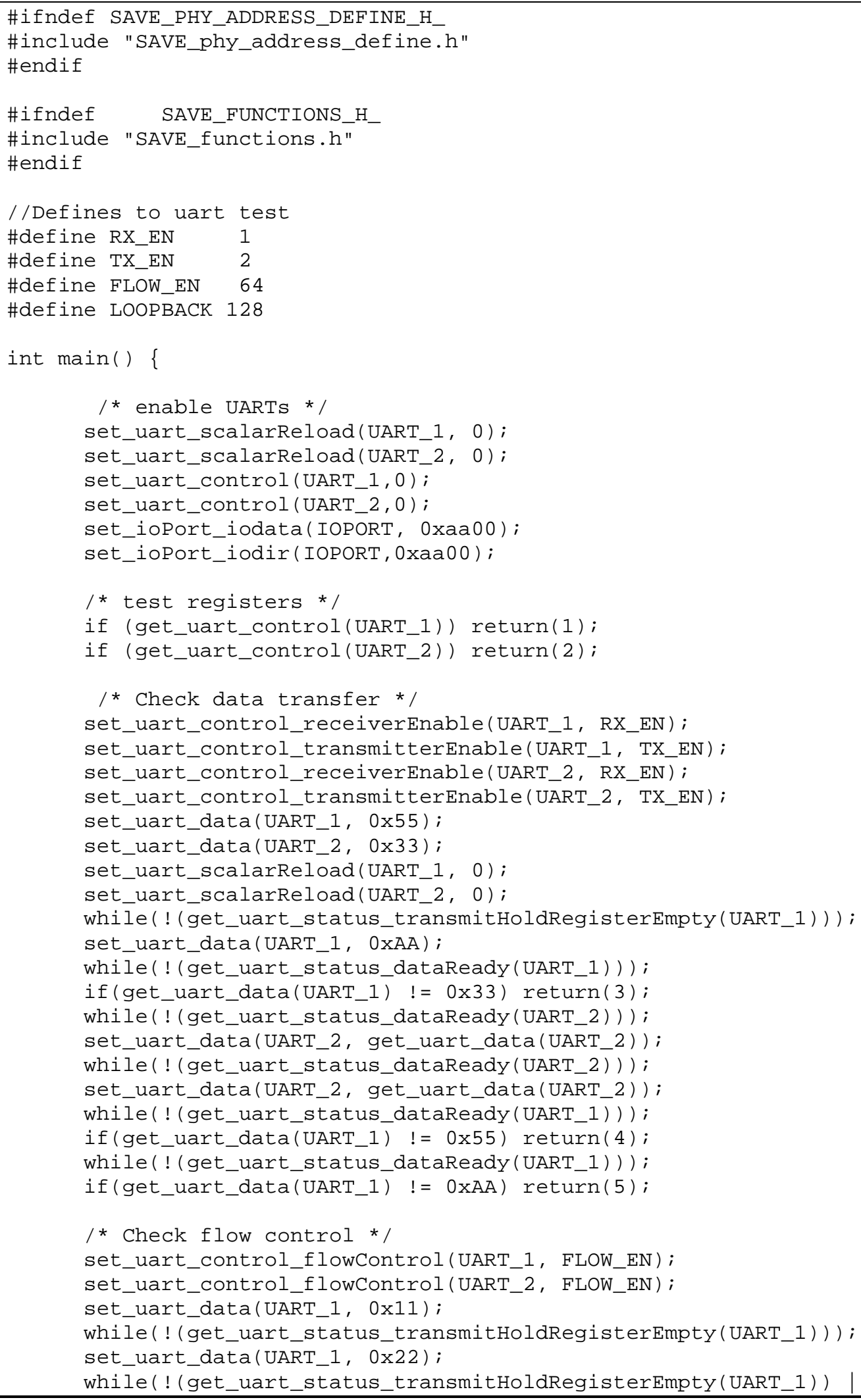




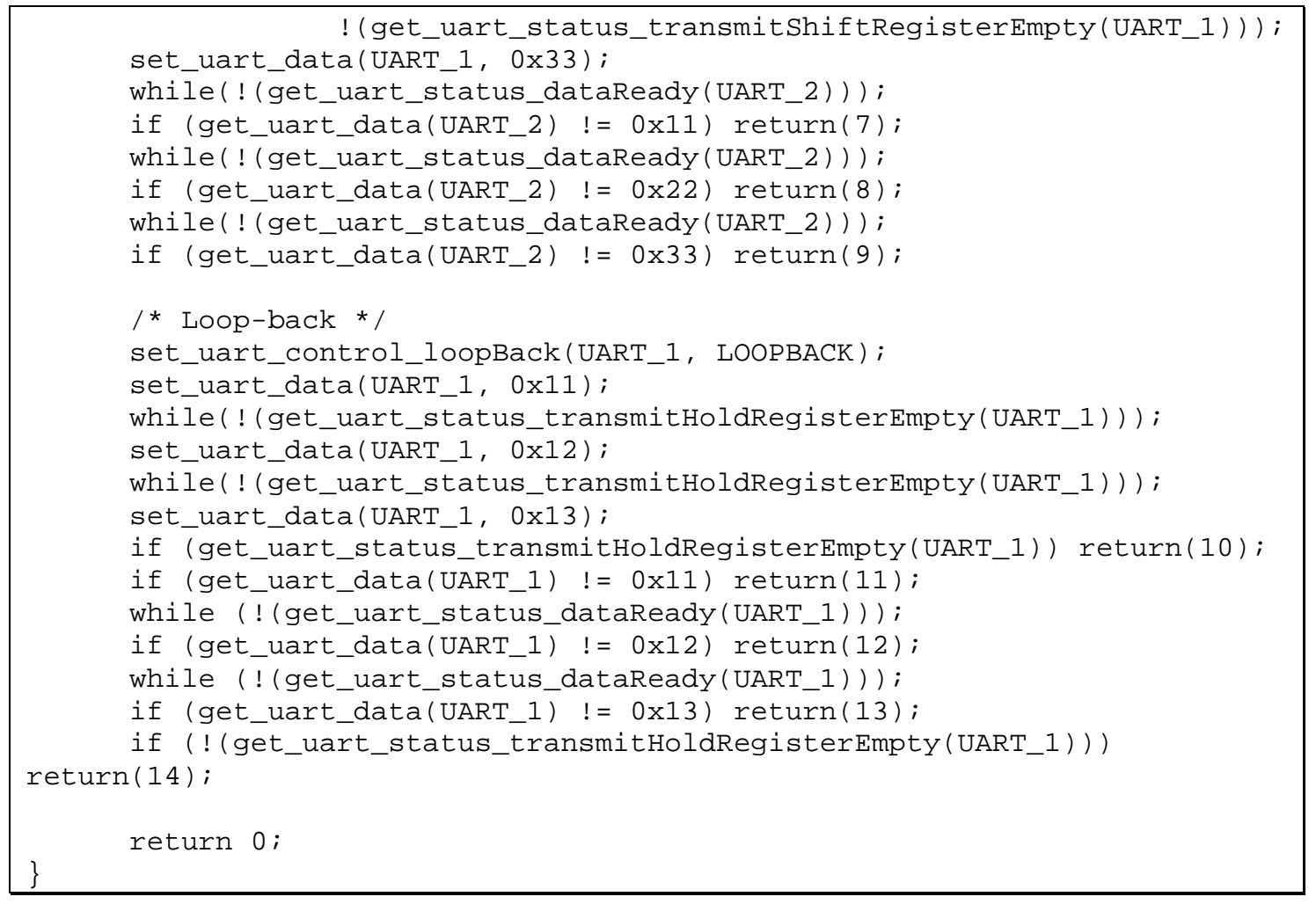


Test cases written without the aid of the HAL functions.

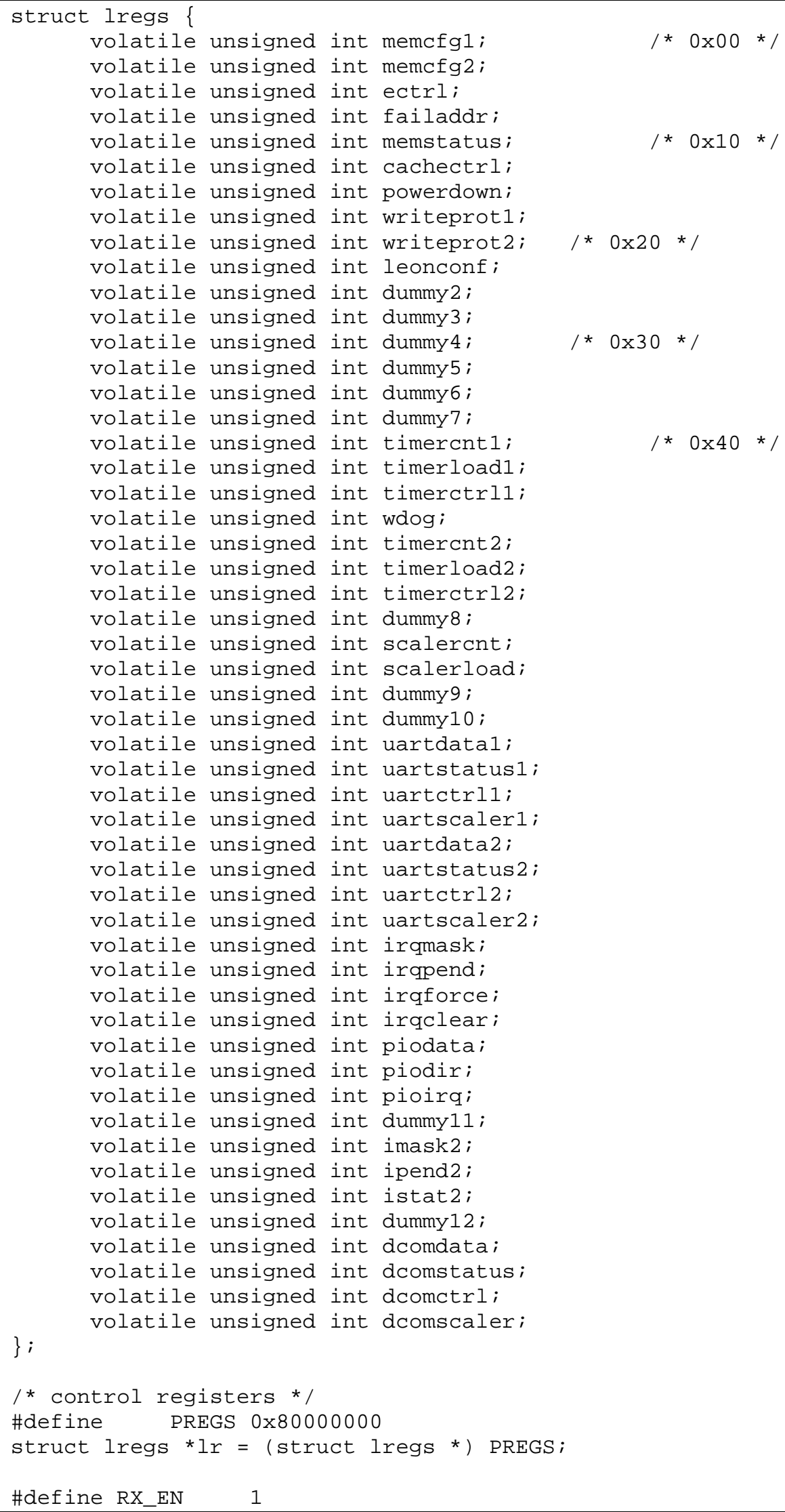




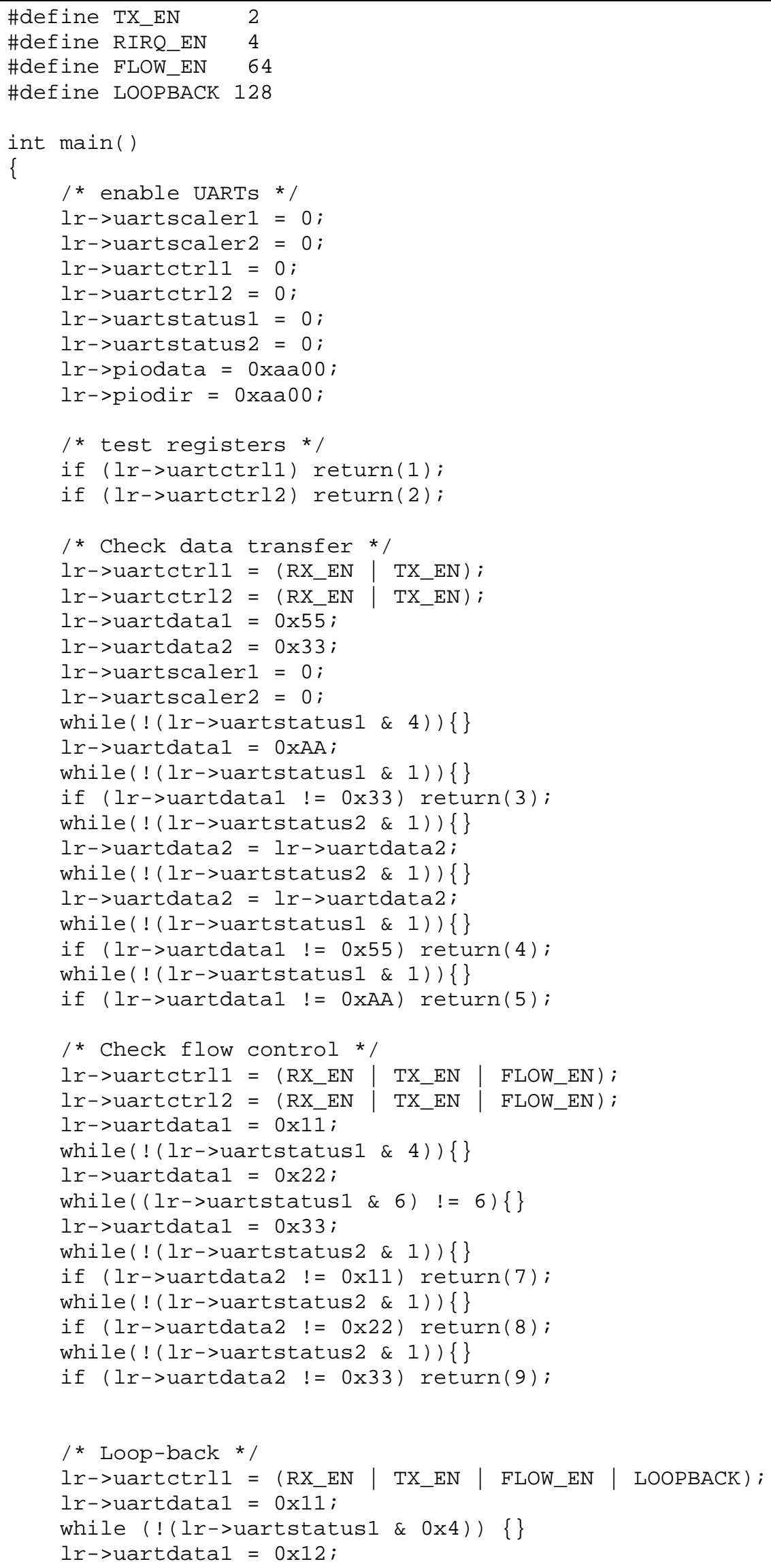


while (! (lr->uartstatus1 \& $0 \times 4))\{\}$

lr->uartdatal = 0x13;

if (lr->uartstatus1 \& $0 \times 4)$ return(10);

if (lr->uartdata1 $!=0 \times 11)$ return(11);

while (! (lr->uartstatus $1 \& 1))\{\}$

if (lr->uartdata1 $!=0 \times 12)$ return(12);

while(! (lr->uartstatus1 \& 1)) \{\}

if (lr->uartdatal $!=0 \times 13)$ return(13);

if (! (lr->uartstatus1\&0x4)) return(14);

return 0 ;

\} 